\title{
FRICTIONAL CONTACT OF AN ANISOTROPIC PIEZOELECTRIC PLATE*
}

\author{
Isabel N. Figueiredo ${ }^{1}$ And Georg Stadler ${ }^{2}$
}

\begin{abstract}
The purpose of this paper is to derive and study a new asymptotic model for the equilibrium state of a thin anisotropic piezoelectric plate in frictional contact with a rigid obstacle. In the asymptotic process, the thickness of the piezoelectric plate is driven to zero and the convergence of the unknowns is studied. This leads to two-dimensional Kirchhoff-Love plate equations, in which mechanical displacement and electric potential are partly decoupled. Based on this model numerical examples are presented that illustrate the mutual interaction between the mechanical displacement and the electric potential. We observe that, compared to purely elastic materials, piezoelectric bodies yield a significantly different contact behavior.
\end{abstract}

Mathematics Subject Classification. 74K20, 78M35, 74M15, 74M10, 74F15

Received April 13, 2007. Revised November 7, 2007.

Published online March 6, 2008.

\section{INTRODUCTION}

The generation of electric charges in certain crystals when subjected to mechanical force was discovered in 1880 by Pierre and Jacques Curie and is nowadays known as piezoelectric effect (or direct piezoelectric effect). The inverse phenomenon, that is, the generation of mechanical stress and strain in crystals when subjected to electric fields is called inverse piezoelectric effect and was predicted in 1881 by Lippmann (see [17]). Piezoelectric materials are solids exhibiting this kind of interaction between mechanical and electric properties. This provides them with sensor (direct effect) and actuator (inverse effect) capabilities making them extremely useful in a wide range of practical applications in aerospace, mechanical, electrical, civil and biomedical engineering (see [29]). In many of these applications, additionally contact phenomena can occur or may be used on purpose, e.g., for measurement devices.

The aim of this paper is to derive, mathematically justify and numerically study a new bi-dimensional model for the equilibrium state of an anisotropic piezoelectric thin plate that is possibly in frictional contact with a rigid foundation. The derivation of the reduced (or lower-dimensional) model is done using an asymptotic procedure. It will turn out that the resulting equations are defined in the middle plane of the plate.

\footnotetext{
Keywords and phrases. Contact, friction, asymptotic analysis, anisotropic material, piezoelectricity, plate.

* This work is partially supported by the project "Mathematical Analysis of Piezoelectric Problems" (POCTI/MAT/59502/2004, Fundação para a Ciência e a Tecnologia, Portugal).

1 Centro de Matemática da Universidade de Coimbra (CMUC), Department of Mathematics, University of Coimbra, Apartado 3008, 3001-454 Coimbra, Portugal. isabelf@mat.uc.pt

2 Institute for Computational Engineering and Sciences (ICES), University of Texas at Austin, 1 University Station C0200, Austin, TX 78712, USA. georgst@ices.utexas.edu
} 
Let us start with motivating our interest in this problem. It was observed that, if no contact and friction conditions have to be taken into account, for certain problems the mechanical and the electric parts of the equations decouple in an asymptotic process $[8,11,26,28]$ (see $[1,7,23,25,33]$ for related results where only partial or no decoupling occurs). In the presence of contact and friction it is not at all obvious if similar results hold true. We are also interested in numerically studying the behavior of piezoelectric materials, and by these means gain a better understanding for their properties and features.

Asymptotic methods have been widely used to deduce reduced models for plates, shells or rods. For the main ideas and bibliographic references see [3,5,6] for elastic plates, [4] for shells, and [32] for rods. For thin elastic plates, asymptotic analysis applies to the thickness variable and can be briefly summarized as follows: starting with the variational three-dimensional equations for a plate with thickness $h$, these equations are scaled to a domain that is independent of $h$. Then, assuming appropriate scalings for the data and unknowns, one lets $h \rightarrow 0$ and studies the convergence of the unknowns as well as the properties of the limit variables. Rescaling to the original domain results in reduced model equations. For a general theory of asymptotic expansions for variational problems that depend on a small parameter we refer to [21].

In this paper we consider an anisotropic piezoelectric plate whose mechanical displacements are restricted due to possible contact with a rigid insulated foundation. The contact is unilateral (i.e., the contact region is not known in advance) and is modelled by the classical Signorini conditions. For the frictional behavior of the plate, the Tresca friction law is used. The variational formulation of this plate problem is a variational inequality of the second kind, see $[9,18]$. The unknowns are the mechanical displacement and the electric potential. The original, three-dimensional plate is subject to contact and friction on a part of its surface. While in the asymptotic procedure the system equations become two-dimensional, the contact and friction conditions remain similar to contact conditions occurring in three-dimensional elasticity.

Note that for modeling friction in physical applications, often the Coulomb rather than the Tresca friction law is used (see [9]). Since for the numerical realization of Coulomb friction usually a sequence of Tresca friction problems is used, Tresca friction is not only of theoretical but also practical relevance. In our numerical study, such a sequence of Tresca problems is used to solve a problem with Coulomb friction.

In the literature, several authors deal with asymptotic models for piezoelectric structures. We mention [26] for piezoelectric plates including magnetic effects, [28] for piezoelectric thin plates with homogeneous isotropic elasticity coefficients, [8,11,12,23,25,33] for anisotropic piezoelectric plates and rods, [7] for geometrically nonlinear thin piezoelectric shells, and [27] for the modelling of eigenvalue problems for thin piezoelectric shells. However, these papers do not take into account the effects of possible contact or friction with a rigid foundation; nevertheless for elastic rods and shells, one finds asymptotic frictionless contact models in [32] and [20], respectively. On the other hand, there are papers dealing with contact and friction of piezoelectric materials that do not use an asymptotic procedure to reduce the model; we refer to [22], where two different variational formulations for the modelling of unilateral frictionless contact are established as well as [2] for primal and dual formulations of frictional contact problems. In [30,31], mathematical analysis of frictional contact problems with piezoelectric materials can be found; for error estimates and numerical simulations we refer to [15]. In all of the above references either none or only few numerical simulations can be found.

The main contributions of this paper are twofold: Firstly, the application of the asymptotic method to the variational inequality of the second kind that describes the anisotropic piezoelectric plate. Due to the presence of friction and contact conditions, the convergence proof in the asymptotic procedure is significantly more involved than in the unconstrained case (see $[11,28]$ ). Our second main contribution is the numerical study of the limit problem taking into account contact and friction. These conditions are similar to three-dimensional elasticity contact problems, where their numerical treatment is known to be a challenging task.

We finish this introduction with a sketch of the structure of this paper. In the next section, the threedimensional plate model is described. In Section 3, we apply the asymptotic analysis and prove strong convergence of the unknowns. Finally, in Section 4 we report on numerical tests for the asymptotic equations. 


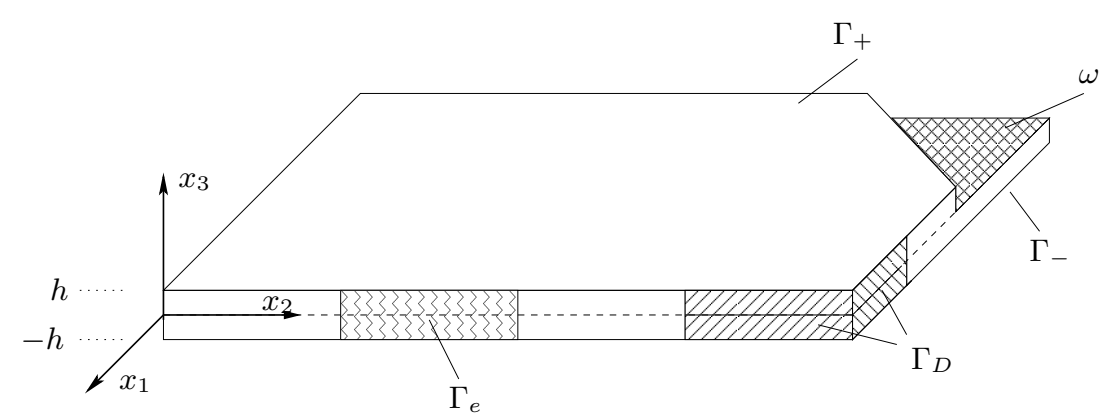

FiguRE 1. Three-dimensional plate $\Omega$ with rectangular middle plane $\omega$ and thickness $2 h$ (note that $\Gamma_{s}=(\partial \omega \times(-h, h)) \backslash \Gamma_{e}$ and $\left.\Gamma_{1}=(\partial \omega \times(-h, h)) \backslash \Gamma_{D}\right)$.

\section{The 3D PLATE PROBLEM}

Notations and geometry. Let $\omega \subset \mathbb{R}^{2}$ be a bounded domain with Lipschitz continuous boundary $\partial \omega, \gamma_{0}$, $\gamma_{e}$ subsets of $\partial \omega$ with meas $\left(\gamma_{0}\right)>0$. We denote $\gamma_{1}:=\partial \omega \backslash \gamma_{0}, \gamma_{s}:=\partial \omega \backslash \gamma_{e}$. For $0<h \ll 1$, we consider $\Omega=\omega \times(-h, h)$ a thin plate with middle plane $\omega$ and thickness $2 h$ and the boundary sets

$$
\begin{aligned}
& \Gamma_{+}=\omega \times\{+h\}, \quad \Gamma_{-}=\omega \times\{-h\}, \quad \Gamma_{ \pm}=\Gamma_{+} \cup \Gamma_{-} \text {, } \\
& \Gamma_{D}=\gamma_{0} \times(-h, h), \quad \Gamma_{1}=\gamma_{1} \times(-h, h), \quad \Gamma_{N}=\Gamma_{1} \cup \Gamma_{+}, \\
& \Gamma_{s}=\gamma_{s} \times(-h, h), \quad \Gamma_{e}=\gamma_{e} \times(-h, h) .
\end{aligned}
$$

For a schematic visualization of $\Omega$ with the boundary sets (2.1) see Figure 1.

We consider different disjoint partitions $\Gamma_{e N}, \Gamma_{e D}$ of the boundary $\partial \Omega$. The splitting correspond to different electric boundary conditions and are thus denoted by $(e b c i)$ for $i=1,2,3$ :

$$
\begin{array}{llll}
(e b c 1): & \Gamma_{e N}=\Gamma_{s} & \text { and } & \Gamma_{e D}=\Gamma_{ \pm} \cup \Gamma_{e} \\
(e b c 2): & \Gamma_{e N}=\Gamma_{s} \cup \Gamma_{+} & \text {and } & \Gamma_{e D}=\Gamma_{-} \cup \Gamma_{e} \\
(e b c 3): & \Gamma_{e N}=\Gamma_{s} \cup \Gamma_{-} & \text {and } & \Gamma_{e D}=\Gamma_{+} \cup \Gamma_{e} .
\end{array}
$$

Note that in all partitions, the set $\Gamma_{e D}$ (where we will assume given Dirichlet data) contains $\Gamma_{-}$or $\Gamma_{+}$. The case that neither on $\Gamma_{-}$nor on $\Gamma_{+}$Dirichlet data for the electric potential are given requires a slightly different treatment than the one chosen in this paper, see Remark 3.4 on page 165.

Points of $\Omega$ are denoted by $x=\left(x_{1}, x_{2}, x_{3}\right)$, where the first two components $\left(x_{1}, x_{2}\right) \in \omega$ are independent of $h$ and $x_{3} \in(-h, h)$. We denote by $n=\left(n_{1}, n_{2}, n_{3}\right)$ the unit outward normal vector to $\partial \Omega$. Throughout the paper, the Latin indices $i, j, k, l, \ldots$ are taken from $\{1,2,3\}$, while the Greek indices $\alpha, \beta, \ldots$ from $\{1,2\}$. The summation convention with respect to repeated indices is employed, that is, $a_{i} b_{i}=\sum_{i=1}^{3} a_{i} b_{i}$. Moreover we denote by $a \cdot b=a_{i} b_{i}$ the inner product of the vectors $a=\left(a_{i}\right)$ and $b=\left(b_{i}\right)$, by $C e=\left(C_{i j k l} e_{k l}\right)$ the contraction of a fourth order tensor $C=\left(C_{i j k l}\right)$ with a second order tensor $e=\left(e_{k l}\right)$ and by $C e: d=C_{i j k l} e_{k l} d_{i j}$ the inner product of the tensors $C e$ and $d=\left(d_{i j}\right)$. Given a function $\theta(x)$ defined in $\Omega$ we denote by $\partial_{i} \theta=\frac{\partial \theta}{\partial x_{i}}$ its partial derivative with respect to $x_{i}$.

In the sequel, for an open subset $\Upsilon \subset \mathbb{R}^{n}, n=2,3$, we define $\mathcal{D}(\Upsilon)$ to be the space of infinitely often differentiable functions with compact support on $\Upsilon$. We denote by $\mathcal{D}^{\prime}(\Upsilon)$ the dual space of $\mathcal{D}(\Upsilon)$, often called the space of distributions on $\Upsilon$. For $m=1,2$, the Sobolev spaces $H^{m}(\Upsilon)$ are defined by

$$
\begin{aligned}
& H^{1}(\Upsilon)=\left\{v \in L^{2}(\Upsilon): \partial_{i} v \in L^{2}(\Upsilon) \text { for } i=1, \ldots, n\right\}, \\
& H^{2}(\Upsilon)=\left\{v \in L^{2}(\Upsilon): \partial_{i} v, \partial_{i j} v \in L^{2}(\Upsilon) \text { for } i, j=1, \ldots, n\right\},
\end{aligned}
$$


where $L^{2}(\Upsilon)=\left\{v: \Upsilon \rightarrow \mathbb{R}, \int_{\Upsilon}|v|^{2} \mathrm{~d} \Upsilon<\infty\right\}$ and the partial derivatives are interpreted as distributional derivatives. Moreover, for $v \in\left(H^{1}(\Omega)\right)^{3}$ we denote by $v_{n}:=v \cdot n$ and $v_{t}:=v-v_{n} n$ the normal and tangential components of $v$ on the boundary of $\Omega$, respectively. Similarly, for a second order symmetric tensor field $\tau=\left(\tau_{i j}\right) \in\left(L^{2}(\Omega)\right)^{9}$ we denote its normal and tangential components on the boundary of $\Omega$ as $\tau_{n}:=(\tau n) \cdot n$ and $\tau_{t}:=(\tau n)-\tau_{n} n$, respectively. Using the summation convention, this becomes $\tau_{n}:=\tau_{i j} n_{i} n_{j}$ and $\tau_{t}=\left(\tau_{t i}\right)$ where $\tau_{t i}:=\tau_{i j} n_{j}-\tau_{n} n_{i}$. In addition, we denote by $|\cdot|$ the Euclidean norm in $\mathbb{R}^{3}$.

3D plate in frictional contact - classical formulation. We consider a piezoelectric anisotropic plate which in its reference configuration occupies the domain $\Omega$. It is held fixed on $\Gamma_{D}$ and submitted to a mechanical volume force of density $f$ in $\Omega$ and a mechanical surface traction of density $g$ on $\Gamma_{N}$. On its lower face $\Gamma_{-}$it may be in frictional contact with the rigid foundation (which is assumed to be an insulator). We denote by $s: \Gamma_{-} \longrightarrow \mathbb{R}^{+}$the initial gap between the rigid foundation and the boundary $\Gamma_{-}$measured in the direction of the outward unit normal vector $n$. To model the frictional contact we use the classical Signorini contact conditions and the Tresca friction law (see [9]).

We assume that the plate is subject to an electric volume charge of density $r$. Moreover, we suppose given an electric surface charge of density $\theta$ on $\Gamma_{e N}$ and an electric potential equal to $\varphi_{0}$ applied to $\Gamma_{e D}$, where the pair $\Gamma_{e N}, \Gamma_{e D}$ is defined as in one of the cases $(e b c i), i=1,2,3$ above. Note that the lower subscripts $e N$ and $e D$ in $\Gamma_{e N}$ and $\Gamma_{e D}$ refer to electric $(e)$ Neumann $(N)$ and Dirichlet $(D)$ boundary conditions, respectively, while the lower subscripts $N$ and ${ }_{D}$ in $\Gamma_{N}$ and $\Gamma_{D}$ refer to mechanical (Neumann and Dirichlet) boundary conditions.

We now give the classical (i.e., strong) equations defining the mechanical and electric equilibrium state of the plate $\Omega$. The equilibrium is described by the following five groups of equations and boundary conditions, whose unknowns are the mechanical displacement vector $u: \Omega \rightarrow \mathbb{R}^{3}$ and the (scalar) electric potential $\varphi: \Omega \rightarrow \mathbb{R}$.

Mechanical equilibrium equations and boundary conditions

$$
\left[\begin{array}{rrll}
-\operatorname{div} \sigma(u, \varphi) & =f \quad\left(i . e .,-\partial_{j} \sigma_{i j}(u, \varphi)=f_{i}\right) & \text { in } \quad & \\
\sigma(u, \varphi) n & =g & \left(i . e ., \sigma_{i j}(u, \varphi) n_{j}=g_{i}\right) & \text { on } \Gamma_{N} \\
u & =0 & \text { on } \Gamma_{D} .
\end{array}\right.
$$

Maxwell-Gauss equations and electric boundary conditions (ebci), $i=1,2,3$

$$
\left[\begin{array}{rlll}
\operatorname{div} D(u, \varphi) & =r \quad\left(\text { i.e., } \partial_{i} D_{i}(u, \varphi)=r\right) & & \text { in } \Omega \\
D(u, \varphi) n & =\theta \quad\left(i . e ., D_{i}(u, \varphi) n_{i}=\theta\right) & & \text { on } \Gamma_{e N} \\
\varphi & =\varphi_{0} & & \text { on } \Gamma_{e D} .
\end{array}\right.
$$

Constitutive equations

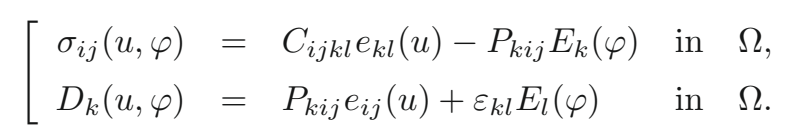

Signorini's contact conditions

$u_{n} \leq s, \quad \sigma_{n} \leq 0, \quad \sigma_{n}\left(u_{n}-s\right)=0 \quad$ on $\quad \Gamma_{-}$.

Tresca's law of friction

$$
\left[\begin{array}{l}
\left|\sigma_{t}\right| \leq q, \quad \text { and } \\
\left|\sigma_{t}\right|<q \quad \Rightarrow \quad u_{t}=0, \\
\left|\sigma_{t}\right|=q \quad \Rightarrow \quad \exists c \geq 0: \quad u_{t}=-c \sigma_{t}
\end{array}\right\} \text { on } \Gamma_{-} .
$$


The mechanical equilibrium equations (2.3a) express the balance of mechanical loads and internal stresses. The electric displacement field $D$ is governed by the Maxwell-Gauss equations (2.3b), and the constitutive equations (2.3c) characterize piezoelectricity. They define the interaction between the stress tensor $\sigma: \Omega \rightarrow \mathbb{R}^{9}$, the electric displacement vector $D: \Omega \rightarrow \mathbb{R}^{3}$, the linear strain tensor $e(u)$ and the electric field vector $E(\varphi)$, the latter two tensors given by

$$
\begin{aligned}
e(u) & =\frac{1}{2}\left(\nabla u+(\nabla u)^{\top}\right) & & \left(\text { i.e., } e_{i j}(u)=\frac{1}{2}\left(\partial_{i} u_{j}+\partial_{j} u_{i}\right)\right) \\
E(\varphi) & =-\nabla \varphi & & \left(\text { i.e., } E_{i}(\varphi)=-\partial_{i} \varphi\right) .
\end{aligned}
$$

In $(2.3 \mathrm{c}), C=\left(C_{i j k l}\right)$ is the elastic fourth order, $P=\left(P_{i j k}\right)$ the piezoelectric third order and $\varepsilon=\left(\varepsilon_{i j}\right)$ is the dielectric second order tensor field. The Signorini law (2.3d) describes the contact behavior of the plate with a rigid foundation. If the plate is not in contact with the rigid foundation (i.e., $u_{n}<s$ ), the normal stress vanishes, i.e., $\sigma_{n}=0$. For $u_{n}=s$, that is, the plate is in contact with the obstacle, the normal stress component $\sigma_{n}$ is nonpositive. These conditions are the complementarity conditions for contact. Finally, the conditions (2.3e) model the frictional behavior of the plate, where $q \geq 0$ is a function representing the prescribed friction bound. Briefly, (2.3e) expresses the fact that on the contact boundary $\Gamma_{-}$the Euclidean norm of the tangential stress component cannot exceed the given friction bound $q$, that slip occurs if this norm equals $q$, and stick if it is smaller than $q$. Moreover, slip can only occur in the negative direction of $\sigma_{t}$. Note that the regions where contact and slip or stick occur are not known a priori. This makes contact problems with friction free boundary problems, which are theoretically and practically challenging.

We assume the following hypotheses on the data

$$
\begin{aligned}
& f \in\left(L^{2}(\Omega)\right)^{3}, g \in\left(L^{2}\left(\Gamma_{N}\right)\right)^{3}, r \in L^{2}(\Omega), \quad \theta \in L^{2}\left(\Gamma_{e N}\right), \\
& \varphi_{0} \in H^{1 / 2}\left(\Gamma_{e D}\right), \quad s \in H^{1 / 2}\left(\Gamma_{-}\right), \quad q \in L^{2}\left(\Gamma_{-}\right),
\end{aligned}
$$

where $\theta=0$ on $\Gamma_{-}$for $(e b c 3)$. Moreover, the tensor fields $C=\left(C_{i j k l}\right), P=\left(P_{i j k}\right)$ and $\varepsilon=\left(\varepsilon_{i j}\right)$ are defined on $\bar{\omega} \times[-1,1]$ for $x=\left(x_{1}, x_{2}, \frac{x_{3}}{h}\right)$. Defining them on the reference plate $\bar{\omega} \times[-1,1]$ makes them independent of $h$ in the transformed variables also used in the next section. The tensors $C_{i j k l}, P_{i j k}, \varepsilon_{i j}$ are assumed to be sufficiently smooth functions that satisfy the following symmetries $C_{i j k l}=C_{j i k l}=C_{k l i j}, P_{i j k}=P_{i k j}, \varepsilon_{i j}=\varepsilon_{j i}$. Moreover, $C$ and $\varepsilon$ are assumed to be coercive, that is there exist $c_{1}, c_{2}>0$ such that

$$
C_{i j k l}(x) M_{k l} M_{i j} \geq c_{1} \sum_{i, j=1}^{3}\left(M_{i j}\right)^{2} \quad \text { and } \quad \varepsilon_{i j}(x) \theta_{i} \theta_{j} \geq c_{2} \sum_{i=1}^{3} \theta_{i}^{2}
$$

for every symmetric $3 \times 3$ real valued matrix $M$, every vector $\theta \in \mathbb{R}^{3}$ and every $x \in \bar{\omega} \times[-1,1]$.

3D-plate in frictional contact - weak formulation. We now give the weak or variational formulation of $(2.3 \mathrm{a})-(2.3 \mathrm{e})$. We define the space of admissible mechanical displacements

$$
V:=\left\{v \in\left(H^{1}(\Omega)\right)^{3}: v_{\mid \Gamma_{D}}=0\right\}
$$

that we endow with the norm $\|v\|_{V}=\|\nabla v\|_{\left(L^{2}(\Omega)\right)^{9}}$, which, due to the Poincaré inequality is equivalent to the usual $H^{1}$-norm. Moreover, we introduce the convex cone

$$
K:=\left\{v \in V: v_{n} \leq s \text { on } \Gamma_{-}\right\}, \text {where } v_{n}=-v_{3},
$$

as well as the space of admissible electric potentials

$$
\Psi:=\left\{\psi \in H^{1}(\Omega): \psi_{\mid \Gamma_{e D}}=0\right\}
$$

in which we use the norm $\|\psi\|_{\Psi}=\|\nabla \psi\|_{\left(L^{2}(\Omega)\right)^{3}}$ (which is also equivalent to the usual $H^{1}$-norm). 
Next, we briefly sketch how the variational formulation of (2.3a)-(2.3e) is obtained. Using the Green formula in (2.3a), we obtain for any $v \in K$

$$
\int_{\Omega} \sigma_{i j} e_{i j}(v-u) \mathrm{d} x-\int_{\partial \Omega} \sigma_{i j} n_{j}\left(v_{i}-u_{i}\right) \mathrm{d} \Gamma_{N}=\int_{\Omega} f_{i}\left(v_{i}-u_{i}\right) \mathrm{d} x .
$$

Since $v=u=0$ on $\Gamma_{D}, \sigma_{i j} n_{j}=g_{i}$ on $\Gamma_{N}, \partial \Omega=\Gamma_{D} \cup \Gamma_{N} \cup \Gamma_{\text {- }}$ and due to

$$
\begin{aligned}
\sigma_{i j} n_{j}\left(v_{i}-u_{i}\right) & =\sigma_{t}\left(v_{t}-u_{t}\right)+\sigma_{n}\left(v_{n}-u_{n}\right) \\
& =\sigma_{t}\left(v_{t}-u_{t}\right)+\sigma_{n}\left(v_{n}-s\right)-\sigma_{n}\left(u_{n}-s\right),
\end{aligned}
$$

(2.5) becomes, using $\sigma_{n}\left(v_{n}-s\right) \geq 0$ and $\sigma_{n}\left(u_{n}-s\right)=0$ on $\Gamma_{-}$,

$$
\int_{\Omega} \sigma_{i j} e_{i j}(v-u) \mathrm{d} x-\int_{\Omega} f_{i}\left(v_{i}-u_{i}\right) \mathrm{d} x-\int_{\Gamma_{N}} g_{i}\left(v_{i}-u_{i}\right) \mathrm{d} \Gamma_{N} \geq \int_{\Gamma_{-}} \sigma_{t}\left(v_{t}-u_{t}\right) \mathrm{d} x .
$$

Adding $j(v)-j(u)$ to both sides of $(2.6)$, where

$$
j(v):=\int_{\Gamma_{-}} q\left|v_{t}\right| \mathrm{d} \Gamma_{-}, \quad \text { with } \quad v_{t}=v-v_{n} n=\left(v_{1}, v_{2}, 0\right),
$$

and using

$$
\int_{\Omega}\left(\sigma_{t}\left(v_{t}-u_{t}\right)+q\left|v_{t}\right|-q\left|u_{t}\right|\right) \mathrm{d} x \geq 0
$$

we obtain

$$
\int_{\Omega} \sigma_{i j} e_{i j}(v-u) \mathrm{d} x+j(v)-j(u)-\int_{\Omega} f_{i}\left(v_{i}-u_{i}\right) \mathrm{d} x-\int_{\Gamma_{N}} g_{i}\left(v_{i}-u_{i}\right) \mathrm{d} \Gamma_{N} \geq 0 .
$$

Next, from (2.3b) we have for any $\psi \in \Psi$

$$
-\int_{\Omega} D_{i} \partial_{i} \psi \mathrm{d} x+\int_{\Gamma_{e N}} \theta \psi \mathrm{d} \Gamma_{e N}=\int_{\Omega} r \psi \mathrm{d} x
$$

where $D_{i} n_{i}=\theta$ on $\Gamma_{e N}$ and $\psi=0$ on $\Gamma_{e D}$ have been used. Summing (2.7) and (2.8), using the constitutive equations (2.3c) and the transformation $\varphi=\bar{\varphi}+\varphi_{0}$, we obtain as weak formulation of (2.3a)-(2.3e) the following elliptic variational inequality of the second kind $[9,13,14,18]$

$$
\left\{\begin{array}{l}
\text { Find } \quad(u, \bar{\varphi}) \in K \times \Psi \quad \text { such that: } \\
b((u, \bar{\varphi}),(v-u, \psi))+j(v)-j(u) \geq l((v-u, \psi)) \quad \forall(v, \psi) \in K \times \Psi
\end{array}\right.
$$

where

$$
\begin{aligned}
b((u, \bar{\varphi}),(v, \psi)):= & \int_{\Omega} C e(u): e(v) \mathrm{d} x+\int_{\Omega} \varepsilon_{i j} \partial_{i} \bar{\varphi} \partial_{j} \psi \mathrm{d} x \\
& +\int_{\Omega} P_{i j k}\left(\partial_{i} \bar{\varphi} e_{j k}(v)-\partial_{i} \psi e_{j k}(u)\right) \mathrm{d} x,
\end{aligned}
$$

and

$$
\begin{aligned}
l((v, \psi)):= & \int_{\Omega} f \cdot v \mathrm{~d} x+\int_{\Gamma_{N}} g \cdot v \mathrm{~d} \Gamma_{N}+\int_{\Omega} r \psi \mathrm{d} x-\int_{\Gamma_{e N}} \theta \psi \mathrm{d} \Gamma_{e N} \\
& -\int_{\Omega} \varepsilon_{i j} \partial_{i} \varphi_{0} \partial_{j} \psi \mathrm{d} x-\int_{\Omega} P_{i j k} \partial_{i} \varphi_{0} e_{j k}(v) \mathrm{d} x .
\end{aligned}
$$


Remark 2.1. Note that

$$
b((v, \psi),(v, \psi))=\int_{\Omega} C e(v): e(v) \mathrm{d} x+\int_{\Omega} \varepsilon_{i j} \partial_{i} \psi \partial_{j} \psi \mathrm{d} x .
$$

Thus, due to $(2.4)$, the bilinear form $b((\cdot, \cdot),(\cdot, \cdot))$ is $V \times \Psi$ - coercive. Since additionally $b((\cdot, \cdot), \cdot, \cdot))$ and the linear form $l((\cdot, \cdot))$ are $V \times \Psi$-continuous, the set $K$ is a nonempty, closed and convex subset of $V$ and the functional $j(\cdot)$ is proper, convex and continuous on $V$, there exists a unique solution $(u, \bar{\varphi})$ of $(2.9)$ (see for instance $[9,13,14,18])$.

\section{Asymptotic ANALYsis}

In this section, we use an asymptotic method, which is mainly due to [3,5,6], to derive two-dimensional plate equations from the three-dimensional system of equations (2.3a)-(2.3e). The principal idea is letting the plate's thickness $h$ tend to zero, after rescaling the 3D variational inequality (2.9) to a fixed reference domain that does not depend on $h$. We investigate the convergence of the unknowns as $h \rightarrow 0$ and analyze the resulting system of equations.

\subsection{Scaling of the $3 D$-equations to a fixed domain}

Here, we redefine the $3 \mathrm{D}$ variational problem (2.9) in the $h$-independent domain $\hat{\Omega}=\omega \times(-1,1)$. To each $x=\left(x_{1}, x_{2}, x_{3}\right) \in \hat{\Omega}$ we associate the element $x=\left(x_{1}, x_{2}, h x_{3}\right) \in \Omega$, through the isomorphism $\pi(x)=$ $\left(x_{1}, x_{2}, h x_{3}\right) \in \Omega$. We consider the subsets defined in (2.1) for the choice $h=1$, that is

$$
\begin{aligned}
\hat{\Gamma}_{ \pm}=\omega \times\{ \pm 1\}, & \hat{\Gamma}_{D}=\gamma_{0} \times(-1,1), \\
\hat{\Gamma}_{1}=\gamma_{1} \times(-1,1), & \hat{\Gamma}_{N}=\hat{\Gamma}_{1} \cup \hat{\Gamma}_{+}, \\
\hat{\Gamma}_{s}=\gamma_{s} \times(-1,1), & \hat{\Gamma}_{e}=\gamma_{e} \times(-1,1),
\end{aligned}
$$

and the disjoint partitions $\hat{\Gamma}_{e N}, \hat{\Gamma}_{e D}$ of $\partial \hat{\Omega}$ defined by consequently replacing $\Gamma$ by $\hat{\Gamma}$ in $(2.2)$.

We denote by $n=\left(n_{1}, n_{2}\right)=\left(n_{\alpha}\right)$ the unit outer normal vector along $\partial \omega$, by $t=\left(t_{1}, t_{2}\right)=\left(t_{\alpha}\right)$, with $t_{1}=-n_{2}$ and $t_{2}=n_{1}$, the unit tangent vector along $\partial \omega$, by $\frac{\partial \theta}{\partial \nu}=\nu_{\alpha} \partial_{\alpha} \theta$ the outer normal derivative of the scalar function $\theta$ along $\partial \omega$. For the asymptotic process we need the data to satisfy the following hypotheses

$$
\begin{aligned}
f_{\alpha} \circ \pi & =h^{2} \hat{f}_{\alpha}, & f_{3} \circ \pi & =h^{3} \hat{f}_{3} \quad \text { in } \hat{\Omega}, \\
g_{\alpha} \circ \pi & =h^{2} \hat{g}_{\alpha}, & g_{3} \circ \pi & =h^{3} \hat{g}_{3} \quad \text { in } \hat{\Gamma}_{1}, \\
g_{\alpha} \circ \pi & =h^{2} \hat{g}_{\alpha}, & g_{3} \circ \pi & =h^{4} \hat{g}_{3} \quad \text { in } \hat{\Gamma}_{+}, \\
\varphi_{0} \circ \pi & =h^{3} \hat{\varphi}_{0}, & r \circ \pi & =h \hat{r} \quad \text { in } \hat{\Omega}, \\
s \circ \pi & =h \hat{s}, & q \circ \pi & =h^{3} \hat{q} \quad \text { in } \hat{\Gamma}_{-}, \\
\theta \circ \pi & =h \hat{\theta} \quad \text { in } \quad \hat{\Gamma}_{s} \cup \hat{\Gamma}_{e}, & \theta \circ \pi & =h^{2} \hat{\theta} \quad \text { in } \hat{\Gamma}_{ \pm} .
\end{aligned}
$$

Above, we assume that $\hat{f}_{\alpha} \in H^{1}(\Omega), \hat{f}_{3} \in L^{2}(\Omega), \hat{g}_{\alpha} \in H^{1}\left(\hat{\Gamma}_{N}\right), \hat{g}_{3} \in L^{2}\left(\hat{\Gamma}_{N}\right), \hat{r} \in L^{2}(\Omega), \hat{\theta} \in L^{2}\left(\hat{\Gamma}_{e N}\right)$,

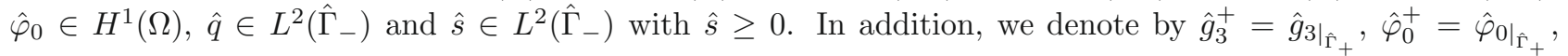
$\hat{\varphi}_{0}^{-}=\hat{\varphi}_{\left.0\right|_{\hat{\Gamma}_{-}}}$and we assume that $\hat{\varphi}_{0}^{+}-\hat{\varphi}_{0}^{-} \in H^{1}(\omega)$. The unknowns are rescaled as follows

$$
u_{\alpha} \circ \pi=h^{2} u_{\alpha}^{h}, \quad u_{3} \circ \pi=h u_{3}^{h} \quad \text { and } \quad \varphi \circ \pi=h^{3} \varphi^{h} \text { in } \hat{\Omega},
$$

and analogous scalings to (3.2) hold for the test functions $v$ and $\psi$ in (2.9). 
At this point, we briefly comment on the choice (3.1)-(3.2) for the scalings of data, unknowns and test functions. For the mechanical forces and displacements we assume the scalings also used in [3] for linearly elastic plates. In [24] it is shown that these are (up to a multiplicative power of $h$ ) "the only possible scalings" that lead to a linear Kirchhoff-Love theory in the asymptotic analysis. Regarding the scalings for the electric variables $\varphi_{0}, r, \theta, \varphi$ and $\psi$ our scalings are chosen such that we are able to compute the limit as $h \rightarrow 0$ of the model (3.6) given below. We also refer to [11,25,28], where scalings as given by (3.1)-(3.2) are used as well. However, different electric scalings have to be used if other electric boundary conditions are chosen, see the remark on page 165 .

The scaled spaces for the admissible mechanical and electric potential displacements are given by

$$
\begin{aligned}
& \hat{V}:=\left\{v \in\left(H^{1}(\hat{\Omega})\right)^{3}: v_{\mid \hat{\Gamma}_{D}}=0\right\}, \\
& \hat{\Psi}:=\left\{\psi \in H^{1}(\hat{\Omega}): \psi_{\mid \hat{\Gamma}_{e D}}=0\right\} .
\end{aligned}
$$

As before, the spaces are endowed with the norms $\|v\|_{\hat{V}}=\|\nabla v\|_{\left(L^{2}(\hat{\Omega})\right)^{9}}$ and $\|\psi\|_{\hat{\Psi}}=\|\nabla \psi\|_{\left(L^{2}(\hat{\Omega})\right)^{3}}$, respectively. The scaled convex cone needed for the Signorini contact conditions is given by

$$
\hat{K}:=\left\{v \in \hat{V}: v_{n}=-v_{3} \leq s \text { on } \hat{\Gamma}_{-}\right\} .
$$

For any $v \in \hat{V}$ we define the second order symmetric tensor field $\kappa^{h}(v)=\left(\kappa_{i j}^{h}(v)\right)$ by

$$
\begin{aligned}
& \kappa_{\alpha \beta}^{h}(v):=e_{\alpha \beta}(v)=\frac{1}{2}\left(\partial_{\beta} v_{\alpha}+\partial_{\alpha} v_{\beta}\right), \\
& \kappa_{\alpha 3}^{h}(v):=\frac{1}{h} e_{\alpha 3}(v)=\frac{1}{2 h}\left(\partial_{3} v_{\alpha}+\partial_{\alpha} v_{3}\right), \\
& \kappa_{33}^{h}(v):=\frac{1}{h^{2}} e_{33}(v)=\frac{1}{h^{2}} \partial_{3} v_{3} .
\end{aligned}
$$

As a consequence of the scalings (3.2) we have for the strain tensors $e(u), e(v)$ with $v \in V$

$$
e(u)=h^{2} \kappa^{h}\left(u^{h}\right) \quad \text { and } \quad e(v)=h^{2} \kappa^{h}(v \circ \pi) \quad \text { with } \quad v \circ \pi \in \hat{V} .
$$

For the stress tensor and the electric displacement vector we obtain the scalings

$$
\sigma_{i j}^{h}\left(u^{h}, \varphi^{h}\right)=h^{-2} \sigma_{i j}(u, \varphi), \quad D_{i}^{h}\left(u^{h}, \varphi^{h}\right)=h^{-2} D_{i}^{h}(u, \varphi),
$$

where

$$
\begin{aligned}
& \sigma_{i j}^{h}\left(u^{h}, \varphi^{h}\right)=C_{i j l m} \kappa_{l m}^{h}\left(u^{h}\right)+h P_{\alpha i j} \partial_{\alpha} \varphi^{h}+P_{3 i j} \partial_{3} \varphi^{h}, \\
& D_{i}^{h}\left(u^{h}, \varphi^{h}\right)=P_{i l m} \kappa_{l m}^{h}\left(u^{h}\right)-h \varepsilon_{i \alpha} \partial_{\alpha} \varphi^{h}-\varepsilon_{i 3} \partial_{3} \varphi^{h} .
\end{aligned}
$$

Weak formulation of the 3D scaled plate problem. Using the above scalings and assumptions (3.1) on the data, we obtain for each $h>0$ a problem on the fixed domain $\hat{\Omega}$ that is equivalent to (2.9):

$$
\left\{\begin{array}{l}
\text { Find } \quad\left(u^{h}, \bar{\varphi}^{h}\right) \in \hat{K} \times \hat{\Psi} \quad \text { such that: } \\
b^{h}\left(\left(\kappa^{h}\left(u^{h}\right), \bar{\varphi}^{h}\right),\left(\kappa^{h}(v)-\kappa^{h}\left(u^{h}\right), \psi\right)\right)+j(v)-j\left(u^{h}\right) \geq l^{h}\left(\left(v-u^{h}, \psi\right)\right), \quad \forall(v, \psi) \in \hat{K} \times \hat{\Psi},
\end{array}\right.
$$


where $\bar{\varphi}^{h}=\varphi^{h}-\hat{\varphi}_{0}$, and for $\kappa, \vartheta$ in $\left(L^{2}(\hat{\Omega})\right)^{9}$ and $\varphi, \psi$ in $\Psi$,

$$
\begin{aligned}
& b^{h}((\kappa, \varphi),(\vartheta, \psi)):=\left\{\begin{array}{l}
\int_{\hat{\Omega}} C \kappa: \vartheta \mathrm{d} x+\int_{\hat{\Omega}} \varepsilon_{33} \partial_{3} \varphi \partial_{3} \psi \mathrm{d} x+\int_{\hat{\Omega}} P_{3 j k}\left(\partial_{3} \varphi \vartheta_{j k}-\partial_{3} \psi \kappa_{j k}\right) \mathrm{d} x \\
+h \int_{\hat{\Omega}} \varepsilon_{3 \alpha}\left(\partial_{\alpha} \varphi \partial_{3} \psi+\partial_{3} \varphi \partial_{\alpha} \psi\right) \mathrm{d} x+h \int_{\hat{\Omega}} P_{\alpha j k}\left(\partial_{\alpha} \varphi \vartheta_{j k}-\partial_{\alpha} \psi \kappa_{j k}\right) \mathrm{d} x \\
+h^{2} \int_{\hat{\Omega}} \varepsilon_{\alpha \beta} \partial_{\alpha} \varphi \partial_{\beta} \psi \mathrm{d} x,
\end{array}\right. \\
& j(v):=\int_{\hat{\Gamma}_{-}} \hat{q}\left|v_{t}\right| \mathrm{d} \hat{\Gamma}_{-} \quad \text { with } \quad v_{t}:=v-v_{n} n=\left(v_{1}, v_{2}, 0\right) \text {, } \\
& l^{h}((v, \psi)):=\left\{\begin{array}{l}
\int_{\hat{\Omega}} \hat{f} \cdot v \mathrm{~d} x+\int_{\hat{\Gamma}_{N}} \hat{g} \cdot v \mathrm{~d} \hat{\Gamma}_{N}+\int_{\hat{\Omega}} \hat{r} \psi \mathrm{d} x-\int_{\hat{\Gamma}_{N}} \hat{\theta} \psi \mathrm{d} \hat{\Gamma}_{e N}-\int_{\hat{\Omega}} \varepsilon_{33} \partial_{3} \hat{\varphi}_{0} \partial_{3} \psi \mathrm{d} x \\
-h \int_{\hat{\Omega}} \varepsilon_{\alpha 3}\left(\partial_{\alpha} \hat{\varphi}_{0} \partial_{3} \psi+\partial_{3} \varphi_{0} \partial_{\alpha} \psi\right) \mathrm{d} x-h^{2} \int_{\hat{\Omega}} \varepsilon_{\alpha \beta} \partial_{\alpha} \hat{\varphi}_{0} \partial_{\beta} \psi \mathrm{d} x \\
-\int_{\hat{\Omega}} P_{3 i j} \partial_{3} \hat{\varphi}_{0} \kappa_{i j}^{h}(v) \mathrm{d} x-h \int_{\hat{\Omega}} P_{\alpha i j} \partial_{\alpha} \hat{\varphi}_{0} \kappa_{i j}^{h}(v) \mathrm{d} x .
\end{array}\right.
\end{aligned}
$$

Using the relation (3.3) between $\kappa^{h}(v)$ and $v$, in the sequel (mainly in the proof of Th. 3.1), we abbreviate

$$
b^{h}\left(\left(\kappa^{h}\left(u^{h}\right), \varphi\right),\left(\kappa^{h}(v), \psi\right)\right) \quad \text { by } \quad b^{h}\left(\left(u^{h}, \varphi\right),(v, \psi)\right) .
$$

In contrast to (2.9), where the dependence on the parameter $h$ is implicit (by means of the domain $\Omega$ ), problem (3.6) now depends explicitly on $h$, but is defined on a domain independent of $h$.

\subsection{Convergence as $\boldsymbol{h} \rightarrow \mathbf{0}^{+}$}

The aim of this section is to study the limit behavior of the sequences $\left(u^{h}\right)$ and $\left(\varphi^{h}\right)$ as $h \rightarrow 0^{+}$. We are able to prove strong convergence of these sequences and give a limit problem that characterizes these limits. It will turn out that the limit displacement is an element of $\hat{V}_{K L}$, the Kirchhoff-Love mechanical displacement space defined by (see also [3])

$$
\begin{aligned}
\hat{V}_{K L}:= & \left\{v=\left(v_{1}, v_{2}, v_{3}\right) \in\left(H^{1}(\hat{\Omega})\right)^{3}: v_{\left.\right|_{\hat{\Gamma}_{D}}}=0, e_{i 3}(v)=0\right\} \\
= & \left\{v=\left(v_{1}, v_{2}, v_{3}\right) \in\left(H^{1}(\hat{\Omega})\right)^{3}: \exists \eta=\left(\eta_{1}, \eta_{2}, \eta_{3}\right) \in\left(H^{1}(\omega)\right)^{2} \times H^{2}(\omega), \partial_{\nu} \eta_{3_{\mid \gamma_{0}}}=0,\right. \\
& \left.\eta_{1_{\mid \gamma_{0}}}=\eta_{2_{\mid \gamma_{0}}}=\eta_{3_{\mid \gamma_{0}}}=0, v_{\alpha}(x)=\eta_{\alpha}\left(x_{1}, x_{2}\right)-x_{3} \partial_{\alpha} \eta_{3}\left(x_{1}, x_{2}\right), v_{3}(x)=\eta_{3}\left(x_{1}, x_{2}\right)\right\} .
\end{aligned}
$$

Moreover, $\hat{\Psi}_{l}$ and $\hat{\Psi}_{l 0}$ are the spaces for the admissible electric potentials defined by

$$
\begin{aligned}
& \hat{\Psi}_{l}:=\left\{\psi \in L^{2}(\hat{\Omega}): \partial_{3} \psi \in L^{2}(\hat{\Omega})\right\} \quad \text { and } \quad \hat{\Psi}_{l 0}:=\left\{\psi \in L^{2}(\hat{\Omega}): \partial_{3} \psi \in L^{2}(\hat{\Omega}), \psi_{\mid S}=0\right\}, \\
& \text { where } \quad S=\left\{\begin{array}{l}
\hat{\Gamma}_{ \pm} \text {for }(e b c 1), \\
\hat{\Gamma}_{-} \text {for }(e b c 2), \\
\hat{\Gamma}_{+} \text {for }(e b c 3) .
\end{array}\right.
\end{aligned}
$$

As usual, $\hat{V}_{K L}$ is endowed with the norm $\|v\|_{\hat{V}_{K L}}:=\left\|e_{\alpha \beta}(v)\right\|_{\left(L^{2}(\hat{\Omega})\right)^{4}}$, which is equivalent to the $\left(H^{1}(\hat{\Omega})\right)^{3}$ norm for elements in $\hat{V}_{K L}$, see [3]. For the space $\hat{\Psi}_{l}$ we use the norm $\|\psi\|_{\hat{\Psi}_{l}}:=\left(\|\psi\|_{L^{2}(\hat{\Omega})}^{2}+\left\|\partial_{3} \psi\right\|_{L^{2}(\hat{\Omega})}^{2}\right)^{1 / 2}$. Finally, $\hat{\Psi}_{l 0}$ is endowed with the norm $\|\psi\|_{\hat{\Psi}_{l 0}}:=\left\|\partial_{3} \psi\right\|_{L^{2}(\hat{\Omega})}$, which is equivalent to the norm defined in $\hat{\Psi}_{l}$ for elements in $\hat{\Psi}_{l 0}$. 
Theorem 3.1. As $h \rightarrow 0^{+}$, the sequence $\left\{\left(u^{h}, \varphi^{h}\right)\right\}_{h}$ converges strongly to $\left(u^{\star}, \varphi^{\star}\right) \in \hat{V}_{K L} \times \hat{\Psi}_{l}$. This limit pair is characterized as the unique solution of the variational problem

$$
\left\{\begin{array}{l}
\text { Find } \quad\left(u^{\star}, \varphi^{\star}\right) \in \hat{V}_{K L} \cap \hat{K} \times \hat{\Psi}_{l} \quad \text { such that: } \\
a\left(\left(u^{\star}, \varphi^{\star}\right),\left(v-u^{\star}, \psi\right)\right)+j(v)-j\left(u^{\star}\right) \geq l\left(\left(v-u^{\star}, \psi\right)\right) \quad \forall(v, \psi) \in \hat{V}_{K L} \cap \hat{K} \times \hat{\Psi}_{l 0}, \\
\varphi^{\star}=\hat{\varphi}_{0}, \text { on } S
\end{array}\right.
$$

where $S \subset \partial \Omega$ is defined in $(3.8 \mathrm{~b})$ and $a(\cdot, \cdot)$ and $l(\cdot)$ are given by

$$
\begin{aligned}
& a\left(\left(u^{\star}, \varphi^{\star}\right),(v, \psi)\right):= \int_{\hat{\Omega}} A_{\alpha \beta \gamma \rho} e_{\alpha \beta}\left(u^{\star}\right) e_{\gamma \rho}(v) \mathrm{d} x+\int_{\hat{\Omega}} p_{33} \partial_{3} \varphi^{\star} \partial_{3} \psi \mathrm{d} x \\
&-\int_{\hat{\Omega}} p_{3 \alpha \beta}\left(e_{\alpha \beta}\left(u^{\star}\right) \partial_{3} \psi-e_{\alpha \beta}(v) \partial_{3} \varphi^{\star}\right) \mathrm{d} x, \\
& l((v, \psi)):=\int_{\hat{\Omega}} \hat{f} \cdot v \mathrm{~d} x+\int_{\hat{\Gamma}_{N}} \hat{g} \cdot v \mathrm{~d} \hat{\Gamma}_{N}+\int_{\hat{\Omega}} \hat{r} \psi \mathrm{d} x-\int_{\hat{\Gamma}_{e}} \hat{\theta} \psi \mathrm{d} \hat{\Gamma}_{N},
\end{aligned}
$$

where $A_{\alpha \beta \gamma \rho}, p_{3 \alpha \beta}$ and $p_{33}$ are modified material parameters (see Part A of the Appendix for details).

Proof. For convenience of the reader, we split the proof into five steps.

Step 1. Existence of weak limits $u^{\star}, \kappa^{\star}$ and $\varphi^{\star}$ of subsequences of $\left(u^{h}\right),\left(\kappa^{h}\left(u^{h}\right)\right)$ and $\left(\varphi^{h}\right)$, respectively. We first choose $(v, \varphi) \in \hat{K} \times \hat{\Psi}$ in (3.6) such that $v_{\alpha}=u_{\alpha}^{h}, v_{3}=0$ and $\psi=-\bar{\varphi}^{h}$. This results in $j(v)-j\left(u^{h}\right)=0$ and thus (3.6) becomes

$$
b^{h}\left(\left(u^{h}, \bar{\varphi}^{h}\right),\left(\left(0,0, u_{3}^{h}\right), \bar{\varphi}^{h}\right)\right) \leq l^{h}\left(\left(\left(0,0, u_{3}^{h}\right), \bar{\varphi}^{h}\right)\right) .
$$

Next, choosing $(v, \varphi) \in \hat{K} \times \hat{\Psi}$ as $v_{\alpha}=-u_{\alpha}^{h}, v_{3}=u_{3}^{h}$ and $\psi=-2 \bar{\varphi}^{h}$, again $j(v)-j\left(u^{h}\right)=0$ and from (3.6)

$$
b^{h}\left(\left(u^{h}, \bar{\varphi}^{h}\right),\left(\left(-2 u_{1}^{h},-2 u_{2}^{h}, 0\right),-2 \bar{\varphi}^{h}\right)\right) \geq l^{h}\left(\left(\left(-2 u_{1}^{h},-2 u_{2}^{h}, 0\right),-2 \bar{\varphi}^{h}\right)\right),
$$

which is equivalent to

$$
b^{h}\left(\left(u^{h}, \bar{\varphi}^{h}\right),\left(\left(u_{1}^{h}, u_{2}^{h}, 0\right), \bar{\varphi}^{h}\right)\right) \leq l^{h}\left(\left(\left(u_{1}^{h}, u_{2}^{h}, 0\right), \bar{\varphi}^{h}\right)\right) .
$$

Adding (3.11) and (3.12) results in

$$
b^{h}\left(\left(u^{h}, \bar{\varphi}^{h}\right),\left(u^{h}, \bar{\varphi}^{h}\right)\right) \leq l^{h}\left(\left(u^{h}, \bar{\varphi}^{h}\right)\right)
$$

from which we can deduce

$$
\left\|u^{h}\right\|_{\hat{V}}^{2}+\int_{\hat{\Omega}} \kappa^{h}\left(u^{h}\right): \kappa^{h}\left(u^{h}\right) \mathrm{d} x+\left\|h \partial_{1} \bar{\varphi}^{h}\right\|_{L^{2}(\hat{\Omega})}^{2}+\left\|h \partial_{2} \bar{\varphi}^{h}\right\|_{L^{2}(\hat{\Omega})}^{2}+\left\|\partial_{3} \bar{\varphi}^{h}\right\|_{L^{2}(\hat{\Omega})}^{2}<c,
$$

where $c>0$ is a constant independent of $h$ (see also [28]). Consequently, there are weakly convergent subsequences of $\left(u^{h}\right),\left(\kappa^{h}\left(u^{h}\right)\right)$ and $\left(\varphi^{h}\right)$ with

$$
\begin{array}{rlrl}
u^{h} & \rightarrow u^{\star} & & \text { in } \quad\left(H^{1}(\hat{\Omega})\right)^{3}, \\
\kappa^{h}\left(u^{h}\right) & \rightarrow \kappa^{\star} & & \text { in } \quad\left(L^{2}(\hat{\Omega})\right)^{9}, \\
\bar{\varphi}^{h} & \rightarrow \bar{\varphi}^{\star}=\varphi^{\star}-\hat{\varphi}_{0} & \text { in } \quad L^{2}(\hat{\Omega}), \\
\varphi^{h} & \rightarrow \varphi^{\star} & \text { in } \quad L^{2}(\hat{\Omega}), \\
\left(h \partial_{1} \varphi^{h}, h \partial_{2} \varphi^{h}, \partial_{3} \varphi^{h}\right) & \rightarrow\left(0,0, \partial_{3} \varphi^{\star}\right) & & \text { in } \quad\left(L^{2}(\hat{\Omega})\right)^{3} .
\end{array}
$$


The first two weak convergences follow directly from (3.13). The existence of the third and fourth weak limit in (3.14) follows from (3.13) using the fact that

$$
\bar{\varphi}^{h}\left(x_{1}, x_{2}, x_{3}\right)=\left\{\begin{array}{l}
\int_{-1}^{x_{3}} \partial_{3} \bar{\varphi}^{h}\left(x_{1}, x_{2}, y_{3}\right) \mathrm{d} y_{3} \quad \text { for } S=\hat{\Gamma}_{ \pm} \text {or } S=\hat{\Gamma}_{-} \\
-\int_{x_{3}}^{+1} \partial_{3} \bar{\varphi}^{h}\left(x_{1}, x_{2}, y_{3}\right) \mathrm{d} y_{3} \quad \text { for } S=\hat{\Gamma}_{+}
\end{array}\right.
$$

which results in

$$
\left\|\bar{\varphi}^{h}\right\|_{L^{2}(\hat{\Omega})} \leq \sqrt{2}\left\|\partial_{3} \bar{\varphi}^{h}\left(x_{1}, x_{2}, x_{3}\right)\right\|_{L^{2}(\hat{\Omega})} \leq c
$$

with $c>0$ independent of $h$. This implies the $L^{2}(\hat{\Omega})$-boundedness of $\bar{\varphi}^{h}$ and $\varphi^{h}=\bar{\varphi}^{h}+\hat{\varphi}_{0}$. In particular, $\varphi^{\star}=\hat{\varphi}_{0}$ on $S$ because $\bar{\varphi}^{h}=0$ on $\hat{\Gamma}_{e} \supseteq S$. Finally, the last convergence in (3.14) is a consequence of (3.13), which implies that

$$
\left(h \partial_{1} \varphi^{h}, h \partial_{2} \varphi^{h}, \partial_{3} \varphi^{h}\right) \rightarrow\left(\vartheta_{1}, \vartheta_{2}, \vartheta_{3}\right) \text { in }\left(L^{2}(\hat{\Omega})\right)^{3} .
$$

The weak convergence of $\varphi^{h}$ to $\varphi^{\star}$ yields $\partial_{i} \varphi^{h} \rightarrow \partial_{i} \varphi^{\star}$ in $L^{2}(\hat{\Omega})$, and thus $\vartheta_{\alpha}=0$ for $\alpha=1,2$, and $\vartheta_{3}=\partial_{3} \varphi^{\star}$.

Moreover, from (3.14) we can also deduce that $u^{\star} \in \hat{V}_{K L}$. In fact, from (3.13) we obtain boundedness of the sequence $\kappa_{i 3}^{h}\left(u^{h}\right)$ in $L^{2}(\hat{\Omega})$. Consequently, $e_{\alpha 3}\left(u^{h}\right)=h \kappa_{\alpha 3}^{h}\left(u^{h}\right)$ and $e_{33}\left(u^{h}\right)=h^{2} \kappa_{33}^{h}\left(u^{h}\right) \rightarrow 0$ strongly in $L^{2}(\hat{\Omega})$. Thus, $e_{i 3}\left(u^{\star}\right):=\frac{1}{2}\left(\partial_{i} u_{3}^{\star}+\partial_{3} u_{i}^{\star}\right)=0$, which implies $u^{\star} \in \hat{V}_{K L}$. We also remark that, appropriate choice of subsequences guarantees that

$$
\kappa_{\alpha \beta}^{\star}=e_{\alpha \beta}\left(u^{\star}\right)=\frac{1}{2}\left(\partial_{\alpha} u_{\beta}^{\star}+\partial_{\beta} u_{\alpha}^{\star}\right)
$$

yielding that the weak limit $\kappa^{\star}$ depends explicitly on $u^{\star}$.

Step 2. Auxiliary results. As a consequence of the weak convergences (3.14) we obtain for arbitrary $(v, \psi) \in$ $\hat{V}_{K L} \times \hat{\Psi}$ that

$$
\begin{aligned}
\lim _{h \rightarrow 0^{+}} b^{h}\left(\left(u^{h}, \varphi^{h}\right),(v, \psi)\right) & :=b^{\star}\left(\left(u^{\star}, \varphi^{\star}\right),(v, \psi)\right), \\
\lim _{h \rightarrow 0^{+}} l^{h}((v, \psi)) & :=l^{\star}((v, \psi)),
\end{aligned}
$$

where for $(v, \psi)$ in $\hat{V}_{K L} \times \hat{\Psi}$

$$
\begin{aligned}
b^{\star}\left(\left(u^{\star}, \varphi^{\star}\right),(v, \psi)\right):= & \int_{\hat{\Omega}} C_{\alpha \beta i j} \kappa_{i j}^{\star} e_{\alpha \beta}(v) \mathrm{d} x+\int_{\hat{\Omega}} \varepsilon_{33} \partial_{3} \varphi^{\star} \partial_{3} \psi \mathrm{d} x \\
& +\int_{\hat{\Omega}} P_{3 \alpha \beta} \partial_{3} \varphi^{\star} e_{\alpha \beta}(v) \mathrm{d} x-\int_{\hat{\Omega}} P_{3 l m} \partial_{3} \psi \kappa_{l m}^{\star} \mathrm{d} x,
\end{aligned}
$$

and

Moreover

$$
\begin{aligned}
l^{\star}((v, \psi)):= & \int_{\hat{\Omega}} \hat{f} \cdot v \mathrm{~d} x+\int_{\hat{\Gamma}_{N}} \hat{g} \cdot v \mathrm{~d} \hat{\Gamma}_{N}+\int_{\hat{\Omega}} \hat{r} \psi \mathrm{d} x-\int_{\hat{\Gamma}_{e N}} \hat{\theta} \psi \mathrm{d} \hat{\Gamma}_{N} \\
& -\int_{\hat{\Omega}} \varepsilon_{33} \partial_{3} \hat{\varphi}_{0} \partial_{3} \psi \mathrm{d} x-\int_{\hat{\Omega}} P_{3 \alpha \beta} \partial_{3} \hat{\varphi}_{0} e_{\alpha \beta}(v) \mathrm{d} x .
\end{aligned}
$$

$$
\lim _{h \rightarrow 0^{+}}\left(j(v)-j\left(u^{h}\right)\right)=j(v)-j\left(u^{\star}\right) .
$$

Step 3. The weak limits $u^{\star}, \kappa^{\star}$ and $\varphi^{\star}$ are also strong limits. Here, it suffices to prove that $\chi^{h}$ strongly converges to $\chi^{\star}$ in $\left(L^{2}(\hat{\Omega})\right)^{12}$, with

$$
\begin{aligned}
& \chi^{h}=\left(\kappa^{h}\left(u^{h}\right), h \partial_{1} \varphi^{h}, h \partial_{2} \varphi^{h}, \partial_{3} \varphi^{h}\right) \in\left(L^{2}(\hat{\Omega})\right)^{12} \\
& \chi^{\star}=\left(\kappa^{\star}, 0,0, \partial_{3} \varphi^{\star}\right) \in\left(L^{2}(\hat{\Omega})\right)^{12} .
\end{aligned}
$$


By the ellipticity and linearity of $b^{h}(.,$.$) , we have$

$$
\begin{aligned}
c\left\|\chi^{h}-\chi^{\star}\right\|_{\left(L^{2}(\hat{\Omega})\right)^{12}}^{2} \leq & b^{h}\left(\left(\kappa^{h}\left(u^{h}\right)-\kappa^{\star}, \varphi^{h}-\varphi^{\star}\right),\left(\kappa^{h}\left(u^{h}\right)-\kappa^{\star}, \varphi^{h}-\varphi^{\star}\right)\right) \\
= & b^{h}\left(\left(\kappa^{h}\left(u^{h}\right), \varphi^{h}\right),\left(\kappa^{h}\left(u^{h}\right), \varphi^{h}\right)\right)+b^{h}\left(\left(\kappa^{\star}, \varphi^{\star}\right),\left(\kappa^{\star}, \varphi^{\star}\right)\right) \\
& -b^{h}\left(\left(\kappa^{h}\left(u^{h}\right), \varphi^{h}\right),\left(\kappa^{\star}, \varphi^{\star}\right)\right)-b^{h}\left(\left(\kappa^{\star}, \varphi^{\star}\right),\left(\kappa^{h}\left(u^{h}\right), \varphi^{h}\right)\right),
\end{aligned}
$$

where $c>0$ is independent of $h$. Replacing $\psi$ by $\psi-\varphi^{h}$ in (3.6), we obtain

$$
b^{h}\left(\left(\kappa^{h}\left(u^{h}\right), \bar{\varphi}^{h}\right),\left(\kappa^{h}(v)-\kappa^{h}\left(u^{h}\right), \psi-\varphi^{h}\right)\right)+j(v)-j\left(u^{h}\right) \geq l^{h}\left(v-u^{h}, \psi-\varphi^{h}\right),
$$

which is, recalling that $\bar{\varphi}^{h}=\varphi^{h}-\hat{\varphi}_{0}$ and noticing that $\left(\kappa^{h}(v)-\kappa^{h}\left(u^{h}\right), \psi-\varphi^{h}\right)=\left(\kappa^{h}(v), \psi\right)-\left(\kappa^{h}\left(u^{h}\right), \varphi^{h}\right)$, equivalent to

$$
\begin{aligned}
b^{h}\left(\left(\kappa^{h}\left(u^{h}\right), \varphi^{h}\right),\left(\kappa^{h}\left(u^{h}\right), \varphi^{h}\right)\right) \leq & b^{h}\left(\left(\kappa^{h}\left(u^{h}\right), \varphi^{h}\right),\left(\kappa^{h}(v), \psi\right)\right)+j(v)-j\left(u^{h}\right) \\
& -\int_{\hat{\Omega}} f \cdot\left(v-u^{h}\right) \mathrm{d} x-\int_{\hat{\Gamma}_{N}} g \cdot\left(v-u^{h}\right) \mathrm{d} \hat{\Gamma}_{N} \\
& -\int_{\hat{\Omega}} r\left(\psi-\varphi^{h}\right) \mathrm{d} x+\int_{\hat{\Gamma}_{e N}} \theta\left(\psi-\varphi^{h}\right) \mathrm{d} \hat{\Gamma}_{e N}
\end{aligned}
$$

for any $v \in \hat{K}$. Thus, considering $v \in \hat{V}_{K L} \cap \hat{K}$ in (3.6), using the weak convergences (3.14) and the limits of Step 2, we derive from (3.19) and (3.20) the estimate

$$
\begin{array}{r}
c \limsup \left\|\chi^{h}-\chi^{\star}\right\|_{\left(L^{2}(\hat{\Omega})\right)^{12}}^{2} \leq b^{\star}\left(\left(u^{\star}, \varphi^{\star}\right),(v, \psi)\right)+j(v)-j(u)-l\left(v-u^{\star}, \psi-\varphi^{\star}\right)-b^{\star}\left(\left(u^{\star}, \varphi^{\star}\right),\left(u^{\star}, \varphi^{\star}\right)\right) \\
=b^{\star}\left(\left(u^{\star}, \varphi^{\star}\right),\left(v-u^{\star}, \psi-\varphi^{\star}\right)\right)+j(v)-j\left(u^{\star}\right)-l\left(v-u^{\star}, \psi-\varphi^{\star}\right),
\end{array}
$$

with $l($.$) as defined in (3.10). Choosing v=u^{\star}$ and $\psi=\varphi^{\star}$ in the above estimate yields

$$
c \lim \sup \left\|\chi^{h}-\chi^{\star}\right\|_{\left(L^{2}(\hat{\Omega})\right)^{12}} \leq 0,
$$

which implies the strong convergence of $\chi^{h}$ to $\chi^{\star}$ in $\left(L^{2}(\hat{\Omega})\right)^{12}$. Due to $\partial_{3}\left(\varphi^{h}-\varphi^{\star}\right) \rightarrow 0$ strongly in $L^{2}(\hat{\Omega})$ and $\varphi^{h}-\varphi^{\star} \in \hat{\Psi}_{l 0}$, we obtain $\varphi^{h}-\varphi^{\star} \rightarrow 0$ strongly in $L^{2}(\hat{\Omega})$ using the equivalence of the norms $\|\cdot\|_{\Psi_{l}}$ and $\|\cdot\|_{\Psi_{l 0}}$ in $\Psi_{l 0}$. Moreover, $e_{i 3}\left(u^{\star}\right)=0, e_{\alpha \beta}\left(u^{\star}\right)=\kappa_{\alpha \beta}^{\star}$ and $\kappa^{h}\left(u^{h}\right) \rightarrow \kappa^{\star}$ strongly in $\left(L^{2}(\hat{\Omega})\right)^{9}$. Thus, we have $e_{\alpha \beta}\left(u^{h}\right) \rightarrow e_{\alpha \beta}\left(u^{\star}\right)$ strongly in $\left(L^{2}(\hat{\Omega})\right)^{9}$, which proves that $u^{h} \rightarrow u^{\star}$ strongly in $\left(H^{1}(\hat{\Omega})\right)^{3}$.

Step 4. Formulas for $\kappa^{\star}=\left(\kappa_{i j}^{\star}\right)$. In (3.17) we already observed that $\kappa_{\alpha \beta}^{\star}=e_{\alpha \beta}\left(u^{\star}\right)$. To obtain formulas for $\kappa_{\alpha 3}^{\star}$ and $\kappa_{33}^{\star}$, we first multiply (3.6) by $h^{2}$ and consider $\psi=0$. Next, we multiply (3.6) by $h$ and consider $v_{3}=u_{3}^{h}$ and $\psi=0$. Due to the strong convergences proved in Step 3, as $h \rightarrow 0^{+}$the limit in the two resulting variational inequalities exists and we obtain

$$
\begin{aligned}
& \int_{\hat{\Omega}}\left(C_{i j 33} \kappa_{i j}^{\star}+P_{333} \partial_{3} \varphi^{\star}\right) \partial_{3}\left(v_{3}-u_{3}^{\star}\right) \mathrm{d} x \geq 0 \forall v_{3} \in H^{1}(\hat{\Omega}), v_{3_{\mid \hat{\Gamma}_{-}}} \geq-s, v_{3_{\mid \hat{\Gamma}_{D}}}=0, \\
& \int_{\hat{\Omega}}\left(C_{i j \alpha 3} \kappa_{i j}^{\star}+P_{3 \alpha 3} \partial_{3} \varphi^{\star}\right) \partial_{3}\left(v_{\alpha}-u_{\alpha}^{\star}\right) \mathrm{d} x \geq 0 \forall v_{\alpha} \in H^{1}(\hat{\Omega}), v_{\alpha_{\mid \hat{\Gamma}_{D}}}=0 .
\end{aligned}
$$


Since $u_{3}^{\star}$ is independent of $x_{3}$, we have $\partial_{3} u_{3}^{\star}=0$ in (3.21a). In (3.21b), we choose $v_{\alpha}:=z_{\alpha}+u_{\alpha}^{\star}$ with $z_{\alpha} \in H^{1}(\hat{\Omega})$ arbitrary with $z_{\alpha_{\mid \hat{\Gamma}_{D}}}=0$. Hence, the inequalities (3.21) become

$$
\begin{aligned}
& \int_{\hat{\Omega}}\left(C_{i j 33} \kappa_{i j}^{\star}+P_{333} \partial_{3} \varphi^{\star}\right) \partial_{3} v_{3} \mathrm{~d} x \geq 0 \forall v_{3} \in H^{1}(\hat{\Omega}), v_{3_{\mid \hat{\Gamma}_{-}}} \geq-s, v_{3_{\mid \hat{\Gamma}_{D}}}=0, \\
& \int_{\hat{\Omega}}\left(C_{i j \alpha 3} \kappa_{i j}^{\star}+P_{3 \alpha 3} \partial_{3} \varphi^{\star}\right) \partial_{3} v_{\alpha} \mathrm{d} x=0 \forall v_{\alpha} \in H^{1}(\hat{\Omega}), \quad v_{\alpha_{\mid \hat{\Gamma}_{D}}}=0 .
\end{aligned}
$$

For arbitrary $\theta \in \mathcal{D}(\hat{\Omega})$ we consider $v_{3}$ in $(3.22 \mathrm{a})$ as

$$
v_{3}\left(x_{1}, x_{2}, x_{3}\right)=\int_{-1}^{x_{3}} \theta\left(x_{1}, x_{2}, t\right) \mathrm{d} t+z_{3}\left(x_{1}, x_{2}\right)
$$

with $z_{3} \in H^{1}(\omega)$ such that $z_{3}\left(x_{1}, x_{2}\right) \geq-s\left(x_{1}, x_{2},-1\right)$ for all $\left(x_{1}, x_{2}\right) \in \omega$. Moreover, we choose $v_{\alpha}$ in $(3.22 \mathrm{~b})$ as

$$
v_{\alpha}\left(x_{1}, x_{2}, x_{3}\right)=\int_{-1}^{x_{3}} \theta\left(x_{1}, x_{2}, t\right) \mathrm{d} t
$$

Then, from (3.22) we obtain

$$
\begin{aligned}
& C_{i j 33} \kappa_{i j}^{\star}+P_{333} \partial_{3} \varphi^{\star}=0 \text { in } L^{2}(\hat{\Omega}), \\
& C_{i j \alpha 3} \kappa_{i j}^{\star}+P_{3 \alpha 3} \partial_{3} \varphi^{\star}=0 \text { in } L^{2}(\hat{\Omega}) .
\end{aligned}
$$

Since $\kappa_{\alpha \beta}^{\star}=e_{\alpha \beta}\left(u^{\star}\right)$, this leads to the formulas

$$
\begin{aligned}
& \kappa_{\alpha 3}^{\star}=-\frac{1}{2} b_{\nu \alpha}\left(a_{\nu \rho \beta} e_{\rho \beta}\left(u^{\star}\right)+c_{\nu} \partial_{3} \varphi^{\star}\right) \\
& \kappa_{33}^{\star}=-\frac{1}{C_{3333}}\left(P_{333} \partial_{3} \varphi^{\star}+C_{33 \alpha \beta} e_{\alpha \beta}\left(u^{\star}\right)\right)+\frac{C_{33 \alpha 3}}{C_{3333}} b_{\nu \alpha}\left(a_{\nu \rho \beta} e_{\rho \beta}\left(u^{\star}\right)+c_{\nu} \partial_{3} \varphi^{\star}\right)
\end{aligned}
$$

where the coefficients $b_{\nu \alpha}, a_{\nu \rho \beta}$ and $c_{\nu}$ are modified material parameters defined in Part A of the Appendix.

Step 5. The limit variational inequality. From the previous Steps 3-4 and (3.5) we directly obtain the following strong $L^{2}(\hat{\Omega})$-convergences, for the scaled stress tensor and the electric displacement vector

$$
\sigma_{i j}^{h}\left(u^{h}, \varphi^{h}\right) \rightarrow \sigma_{i j}^{\star} \quad \text { and } \quad D_{i}^{h}\left(u^{h}, \varphi^{h}\right) \rightarrow D_{i}^{\star}
$$

where

$$
\begin{aligned}
& \sigma_{\alpha \beta}^{\star}=C_{\alpha \beta l m} \kappa_{l m}^{\star}+P_{3 \alpha \beta} \partial_{3} \varphi^{\star} \\
& \left.\sigma_{i 3}^{\star}=C_{i 3 l m} \kappa_{l m}^{\star}+P_{3 i 3} \partial_{3} \varphi^{\star}=0 \quad \text { (because of }(3.23)\right) \\
& D_{i}^{\star}=P_{i l m} \kappa_{l m}^{\star}-\varepsilon_{i 3} \partial_{3} \varphi^{\star} .
\end{aligned}
$$

With (3.24) for $\kappa^{\star}$ we get

$$
\begin{aligned}
& \sigma_{\alpha \beta}^{\star}=A_{\alpha \beta \gamma \rho} e_{\alpha \beta}\left(u^{\star}\right)+p_{3 \alpha \beta} \partial_{3} \varphi^{\star} \\
& D_{i}^{\star}=p_{i \alpha \beta} e_{\alpha \beta}\left(u^{\star}\right)-p_{i 3} \partial_{3} \varphi^{\star},
\end{aligned}
$$

where the coefficients $A_{\alpha \beta \gamma \rho}, p_{i \alpha \beta}$ and $p_{i 3}$ are defined in the Appendix, Part A. 
Using again the strong convergences obtained in Step 3 and $\varphi^{\star}=\bar{\varphi}^{\star}+\hat{\varphi}_{0}$ we can take the limit in (3.6) and obtain the limit variational inequality:

$$
\left\{\begin{array}{l}
\text { Find } \quad\left(u^{\star}, \varphi^{\star}\right) \in \hat{V}_{K L} \cap \hat{K} \times \hat{\Psi}_{l} \quad \text { such that: } \\
b^{\star}\left(\left(u^{\star}, \varphi^{\star}\right),\left(v-u^{\star}, \psi\right)\right)+j(v)-j\left(u^{\star}\right) \geq l\left(\left(v-u^{\star}, \psi\right)\right) \quad \forall(v, \psi) \in \hat{V}_{K L} \cap \hat{K} \times \hat{\Psi}_{l 0}, \\
\varphi^{\star}=\hat{\varphi}_{0} \text { on } S .
\end{array}\right.
$$

Here the linear form $l(\cdot)$ is defined by $(3.10)$ and the bilinear form $b^{\star}(\cdot, \cdot)$ by $(3.18)$. From $(3.25)$ one obtains

$$
b^{\star}\left(\left(u^{\star}, \varphi^{\star}\right),(v, \psi)\right)=\int_{\hat{\Omega}} \sigma_{\alpha \beta}^{\star} e_{\alpha \beta}(v) \mathrm{d} x-\int_{\hat{\Omega}} D_{3}^{\star} \partial_{3} \psi \mathrm{d} x
$$

Using now the equivalent definitions of $\sigma_{\alpha \beta}^{\star}$ and $D_{3}^{\star}$ given in (3.26), the right-hand side of (3.28) turns out to be precisely $a\left(\left(u^{\star}, \varphi^{\star}\right),(v, \psi)\right)$. Hence (3.27) coincides with the limit problem (3.9) and the proof of the theorem is finished. We remark that, the solution of this limit problem is unique if the bilinear form $a(\cdot, \cdot)$ in $(3.9)$ is elliptic in the set $V_{K L} \cap \hat{K} \times \hat{\Psi}_{l 0}$ (cf. [14]). This is, for instance, the case if the material is mechanically monoclinic, that is, $C_{\alpha \beta \gamma 3}=0=C_{\alpha 333}$ (see Th. 3.3 in [11]). One easily verifies that this ellipticity result also holds true for a laminated plate with mechanically monoclinic piezoelectric layers.

Remark 3.2. Note that the proof of the previous theorem is technically more involved than in the case without contact (see $[8,11,28]$ ). In particular, to identify the asymptotic model (3.9) the derivation of explicit formulas for $\kappa^{\star}=\left(\kappa_{i j}^{\star}\right)$ is crucial: in the case without contact weak convergences of the sequences $\left(u^{h}\right),\left(\varphi^{h}\right)$ and $\left(\kappa^{h}\left(u^{h}\right)\right)$ is sufficient, while for the contact case it is necessary to prove the strong convergences (see Step 3). Moreover, in order to derive formulas for $\kappa^{\star}=\left(\kappa_{i j}^{\star}\right)$ one needs to choose appropriate test functions (see Step 4).

\subsection{Rescaling to the original domain}

The limit variational inequality (3.9) can be rescaled to the original plate $\Omega=\omega \times(-h, h)$. In order to do so, let $x_{3} \in(-1,+1)$ and $u_{\alpha}^{\star}=\zeta_{\alpha}^{\star}-x_{3} \partial_{\alpha} \zeta_{3}^{\star}, u_{3}^{\star}=\zeta_{3}^{\star}$ be the components of the Kirchhoff-Love limit displacement $u^{\star}$. The corresponding descaled function $\zeta=\left(\zeta_{1}, \zeta_{2}, \zeta_{3}\right)$ is given by

$$
\zeta_{\alpha}:=h^{2} \zeta_{\alpha}^{\star}, \quad \zeta_{3}:=h \zeta_{3}^{\star} \quad \text { in } \quad \bar{\omega}
$$

which leads to the descaled variables

$$
\begin{aligned}
u_{\alpha}(x) & :=h^{2} u_{\alpha}^{\star}=\zeta_{\alpha}\left(x_{1}, x_{2}\right)-x_{3} \partial_{\alpha} \zeta_{3}\left(x_{1}, x_{2}\right), \\
u_{3}(x) & :=h u_{3}^{\star}\left(x_{1}, x_{2}\right)=\zeta_{3}\left(x_{1}, x_{2}\right), \\
\varphi(x) & :=h^{3} \varphi^{\star}(x),
\end{aligned}
$$

for $x=\left(x_{1}, x_{2}, x_{3}\right) \in \Omega=\omega \times(-h, h)$. Above, $\zeta_{\alpha}$ and $\zeta_{3}$ are in-plane and transverse Kirchhoff-Love displacements, $u_{i}$ is the limit mechanical displacements and $\varphi$ the electric potential inside the plate $\Omega$. The spaces $V_{K L}$, $\Psi_{l}$ and $\Psi_{l 0}$ correspond to the descaled variables defined over $\Omega$ and are given by (3.7)-(3.8) with $\hat{\Omega}$ replaced by $\Omega$ and $\hat{\Gamma}$ by $\Gamma$ in the definition of $S$. 
Plugging the rescaled variables in the limit problem found in Theorem 3.1, we obtain the following rescaled limit problem:

$$
\left\{\begin{array}{l}
\text { Find } \quad(u, \varphi) \in V_{K L} \cap K \times \Psi_{l} \quad \text { such that: } \\
a((u, \varphi),(v-u, \psi))+j(v)-j(u) \geq l((v-u, \psi)), \quad \forall(v, \psi) \in V_{K L} \cap K \times \Psi_{l 0}, \\
\varphi=\varphi_{0}, \quad \text { on } \quad S
\end{array}\right.
$$

where for $u, v$ in $V_{K L}$ and $\varphi, \psi$ in $\Psi$

$$
\begin{aligned}
a((u, \varphi),(v, \psi)):= & \int_{\Omega} A_{\alpha \beta \gamma \rho} e_{\alpha \beta}(u) e_{\gamma \rho}(v) \mathrm{d} x+\int_{\Omega} p_{33} \partial_{3} \varphi \partial_{3} \psi \mathrm{d} x \\
& -\int_{\Omega} p_{3 \alpha \beta}\left(e_{\alpha \beta}(u) \partial_{3} \psi-e_{\alpha \beta}(v) \partial_{3} \varphi\right) \mathrm{d} x
\end{aligned}
$$

and

$$
l((v, \psi)):=\int_{\Omega} f \cdot v \mathrm{~d} x+\int_{\Gamma_{N}} g \cdot v \mathrm{~d} \Gamma_{N}+\int_{\Omega} r \psi \mathrm{d} x-\int_{\Gamma_{e N}} \theta \psi \mathrm{d} \Gamma_{e N} .
$$

\subsection{Decoupling of $u$ and $\varphi$}

The structure of the bilinear form $a(\cdot, \cdot)$, obtained by the asymptotic procedure above allows a certain uncoupling of the mechanical displacement $u$ and the electric potential $\varphi$. This leads to a variational inequality in the mechanical displacement $u$ only, and an explicit formula for the electric potential. This explicit form, which is a second order polynomial with coefficients that depend on the Kirchhoff-Love displacement $u$, obeys a slightly different form for each of the boundary partitions $(e b c 1),(e b c 2),(e b c 3)$ for the electric data.

To derive the decoupling, we choose $v=u$ in the variational inequality (3.29) and obtain

$$
\int_{\Omega}\left(p_{33} \partial_{3} \varphi-p_{3 \alpha \beta} e_{\alpha \beta}(u)\right) \partial_{3} \psi \mathrm{d} x=\int_{\Omega} r \psi \mathrm{d} x-\int_{\Gamma_{e N}} \theta \psi \mathrm{d} \Gamma_{e N}
$$

for all $\psi \in \Psi_{l 0}$. Due to the density of $\Psi_{l 0}$ in $\mathcal{D}(\Omega)$ (see [28]), this yields the following formula for $\partial_{3} \varphi$

$$
\partial_{3} \varphi=\frac{p_{3 \alpha \beta}}{p_{33}}\left(e_{\alpha \beta}(\zeta)-x_{3} \partial_{\alpha \beta} \zeta_{3}\right)-\frac{d}{p_{33}} \quad \text { with } \quad d=P_{3} r+c,
$$

where $c \in \mathcal{D}(\omega)$ and $P_{3} r=\int_{-h}^{x_{3}} r \mathrm{~d} y_{3}$ denotes the antiderivative of $r$ with respect to the thickness variable $x_{3}$. Using this latter formula and one of the boundary conditions (ebci), we obtain explicit formulas for the electric potential. In the case that the electric potential is given on both the upper and lower surface $\Gamma_{-}$and $\Gamma_{+}$, we integrate (3.30) with respect to $x_{3}$. Then, using the given boundary data, we obtain the formula (see also Th. 2.1 in $[8])$

$$
\begin{aligned}
\varphi\left(x_{1}, x_{2}, x_{3}\right) & =\varphi_{0}^{-}\left(x_{1}, x_{2}\right) \\
& +\int_{-h}^{x_{3}}\left(\left(\frac{p_{3 \alpha \beta}}{p_{33}}-\frac{a_{\alpha \beta}}{p_{33}} c_{0}\right) e_{\alpha \beta}(\zeta)-\left(\frac{p_{3 \alpha \beta}}{p_{33}} y_{3}-\frac{b_{\alpha \beta}}{p_{33}} c_{0}\right) \partial_{\alpha \beta} \zeta_{3}+\frac{\left(\varphi_{0}^{+}-\varphi_{0}^{-}+R\right) c_{0}-P_{3} r}{p_{33}}\right) \mathrm{d} y_{3},
\end{aligned}
$$

where

$$
a_{\alpha \beta}:=\int_{-h}^{+h} \frac{p_{3 \alpha \beta}}{p_{33}} \mathrm{~d} x_{3}, \quad b_{\alpha \beta}:=\int_{-h}^{+h} x_{3} \frac{p_{3 \alpha \beta}}{p_{33}} \mathrm{~d} x_{3}, \quad c_{0}=\left(\int_{-h}^{+h} \frac{1}{p_{33}} \mathrm{~d} x_{3}\right)^{-1}, \quad R:=\int_{-h}^{+h} \frac{P_{3} r}{p_{33}} \mathrm{~d} x_{3} .
$$


For the case that the electric potential is either given on $\Gamma_{-}$or $\Gamma_{+}$, i.e., for the cases $(e b c 2)$ and $(e b c 3)$, we plug (3.30) into (3.29) and choose again $v=u$ to obtain

$$
-\int_{\Omega}\left(P_{3} r+c\right) \partial_{3} \psi \mathrm{d} x=\int_{\Omega} r \psi \mathrm{d} x-\int_{\Gamma_{e N}} \theta \psi \mathrm{d} \Gamma_{e N} .
$$

From the Green formula we obtain $\partial_{3}\left(P_{3} r+c\right)=r$ in $\mathcal{D}(\Omega)$ and

$$
-\int_{\Gamma_{e N}}\left(P_{3} r+c\right) n_{3} \psi \mathrm{d} x-\int_{\Gamma_{e D}}\left(P_{3} r+c\right) n_{3} \psi \mathrm{d} x=-\int_{\Gamma_{e N}} \theta \psi \mathrm{d} \Gamma_{e N}
$$

with $n_{3}=0$ on the lateral boundaries $\Gamma_{e}$ and $\Gamma_{s}, n_{3}= \pm 1$ on $\Gamma_{ \pm}$and $\psi=0$ on $\Gamma_{e D}$. Choosing $\psi$ such that $\psi=0$ on the lateral boundary, (3.32) reduces to

$$
\begin{aligned}
\int_{\Gamma_{+}}\left(P_{3} r+c\right) \psi \mathrm{d} \Gamma_{+} & =\int_{\Gamma_{+}} \theta \psi \mathrm{d} \Gamma_{+} \text {for }(e b c 2), \\
-\int_{\Gamma_{-}}\left(P_{3} r+c\right) \psi \mathrm{d} \Gamma_{-} & =\int_{\Gamma_{-}} \theta \psi \mathrm{d} \Gamma_{-} \text {for }(e b c 3) .
\end{aligned}
$$

This implies, for $(e b c 2)$ that $c\left(x_{1}, x_{2}\right)=\left(\theta-P_{3} r\right)\left(x_{1}, x_{2},+h\right)$, and for $(e b c 3)$ that $c\left(x_{1}, x_{2}\right)=\left(-\theta-P_{3} r\right)\left(x_{1}, x_{2}\right.$, $-h$ ). Consequently, integrating (3.30) with $d=P_{3} r+c$ along the thickness variable (from $-h$ to $x_{3}$ for $(e b c 2)$ and from $x_{3}$ to $+h$ for $(e b c 3)$ ), we obtain

$$
\begin{aligned}
& \varphi\left(x_{1}, x_{2}, x_{3}\right)=\varphi_{0}^{-}\left(x_{1}, x_{2}\right)+\int_{-h}^{x_{3}} \frac{p_{3 \alpha \beta}}{p_{33}}\left(e_{\alpha \beta}(\zeta)-y_{3} \partial_{\alpha \beta} \zeta_{3}\right) \mathrm{d} y_{3} \\
&-\int_{-h}^{x_{3}} \frac{P_{3} r\left(x_{1}, x_{2}, y_{3}\right)+\left(\theta-P_{3} r\right)\left(x_{1}, x_{2},+h\right)}{p_{33}} \mathrm{~d} y_{3} \quad \text { for }(e b c 2)
\end{aligned}
$$

and

$$
\begin{aligned}
\varphi\left(x_{1}, x_{2}, x_{3}\right)=\varphi_{0}^{+}\left(x_{1}, x_{2}\right) & -\int_{x_{3}}^{+h} \frac{p_{3 \alpha \beta}}{p_{33}}\left(e_{\alpha \beta}(\zeta)-y_{3} \partial_{\alpha \beta} \zeta_{3}\right) \mathrm{d} y_{3} \\
& +\int_{x_{3}}^{+h} \frac{P_{3} r\left(x_{1}, x_{2}, y_{3}\right)+\left(-\theta-P_{3} r\right)\left(x_{1}, x_{2},-h\right)}{p_{33}} \mathrm{~d} y_{3} \quad \text { for }(e b c 3) .
\end{aligned}
$$

Next, we plug the above explicit forms for the electric potential into (3.29) and choose the electric test function $\psi=0$. This gives an equivalent formulation for the variational inequality (3.29), which is summarized in the next theorem. Now, the mechanical displacement and the electric potential are not coupled in the variational inequality any more. The main advantage of this decoupled formulation is that after solving a variational inequality for the mechanical displacement, one can use an explicit formula for the electric potential.

Theorem 3.3. Let $(u, \varphi) \in V_{K L} \times \Psi_{l}$ be a solution of problem (3.29), where $u_{\alpha}=\zeta_{\alpha}-x_{3} \partial_{\alpha} \zeta_{3}, u_{3}=\zeta_{3}$, and $\zeta=\left(\zeta_{1}, \zeta_{2}, \zeta_{3}\right)$. Then the Kirchhoff-Love mechanical displacement $u \in V_{K L}$ is also characterized as solution of the variational inequality

$$
\left\{\begin{array}{l}
\text { Find } \quad u \in V_{K L} \cap K \quad \text { such that: } \\
a^{e b c i}(u, v-u)+j(v)-j(u) \geq l^{e b c i}(v-u) \quad \forall v \in V_{K L} \cap K,
\end{array}\right.
$$


and the electric potential can be derived a posteriori from (3.31) for (ebc1), (3.33) for (ebc2) and (3.34) for (ebc3). The modified bilinear and linear forms, respectively $a^{\text {ebci }}(\cdot, \cdot)$ and $l^{\text {ebci }}(\cdot)$, are defined by

$$
\begin{aligned}
a^{e b c i}(u, v) & :=\int_{\omega}\left(N_{\alpha \beta}^{e b c i}(u) e_{\alpha \beta}(\eta)+M_{\alpha \beta}^{e b c i}(u) \partial_{\alpha \beta} \eta_{3}\right) \mathrm{d} \omega \\
l^{e b c i}(v) & :=\int_{\Omega} f \cdot v \mathrm{~d} \Omega+\int_{\Gamma_{N}} g \cdot v \mathrm{~d} \Gamma_{N}+l_{e}^{e b c i}(v),
\end{aligned}
$$

where $N_{\alpha \beta}^{e b c i}(u), M_{\alpha \beta}^{e b c i}(u)$ and $l_{e}^{e b c i}($.$) are detailed in Part B of the Appendix.$

Remark 3.4. Let us comment on a different (fourth) choice of electric boundary condition (ebc4) given by

$$
(e b c 4): \quad \Gamma_{e N}=\Gamma_{s} \cup \Gamma_{-} \cup \Gamma_{+} \quad \text { and } \quad \Gamma_{e D}=\Gamma_{e}
$$

where we assume meas $\left(\gamma_{e}\right)>0$. This means that we apply an electric surface charge on both the upper and lower surface of the plate. This case requires a different treatment in the asymptotic analysis: Note that instead of (3.14) we only obtain $u^{h} \rightarrow u^{\star}$ in $\left(H^{1}(\hat{\Omega})\right)^{3}, \kappa^{h}\left(u^{h}\right) \rightarrow \kappa^{\star}$ in $\left(L^{2}(\hat{\Omega})\right)^{9}$ and $\left(h \partial_{1} \varphi^{h}, h \partial_{2} \varphi^{h}, \partial_{3} \varphi^{h}\right) \rightarrow$ $\left(\vartheta_{1}, \vartheta_{2}, \vartheta_{3}\right)$ in $\left(L^{2}(\hat{\Omega})\right)^{3}$. Now, we cannot conclude that $\varphi^{h}, \bar{\varphi}^{h}$ are weakly convergent since $(3.15)$ and consequently (3.16) do not apply. Hence, $\vartheta_{1}, \vartheta_{2}$ are not necessary equal to zero and the limit problem changes considerably. This is in accordance with observations in $[25,26,33]$, where it is shown that for different electric boundary conditions significantly different limit problems may arise. To obtain an easier interpretation for the limit problem in case of $(e b c 4)$, it might be advantageous to consider scalings different from those used in this paper for the electric potential.

Remark 3.5. We now sketch the strong (i.e., the differential) form of the limit problem obtained in Theorem 3.3. This form uses Lagrange multipliers to resolve the contact and friction conditions and is obtained assuming sufficient regularity of $(u, \varphi)$ as well as partial integration. It follows from duality theory [10] that there exist so called multipliers (or dual variables) $(\lambda, \mu) \in\left(H^{2}(\omega)\right)^{\prime} \times\left(L^{2}(\omega)\right)^{2}$ satisfying complementarity conditions (see $(3.39 \mathrm{~d}),(3.39 \mathrm{e})$ below) for $(3.35)$, where $\left(H^{2}(\omega)\right)^{\prime}$ denotes the dual of $H^{2}(\omega)$. Then, $u \in V_{K L} \cap K$ can be written as

$$
a^{e b c i}(u, v)+\int_{\Gamma_{-}} \lambda v_{n} \mathrm{~d} \Gamma_{-}+\int_{\Gamma_{-}} \mu \cdot v_{t} \mathrm{~d} \Gamma_{-}=l^{e b c i}(v), \quad \forall v \in V_{K L}
$$

Choosing test functions $v=\left(\eta_{1}-x_{3} \partial_{1} \eta_{3}, \eta_{2}-x_{3} \partial_{2} \eta_{3}, \eta_{3}\right) \in V_{K L}$ with $\eta_{1}=\eta_{2}=0$ and $\eta_{3} \neq 0$, i.e., $v=$ $\left(-x_{3} \partial_{1} \eta_{3},-x_{3} \partial_{2} \eta_{3}, \eta_{3}\right),(3.36)$ is equivalent to

$$
\begin{aligned}
\int_{\omega} M_{\alpha \beta}^{e b c i}(u) \partial_{\alpha \beta} \eta_{3}-\int_{\Gamma_{-}} \lambda \eta_{3} \mathrm{~d} \Gamma_{-}+\int_{\Gamma_{-}} \mu_{\alpha}\left(-x_{3} \partial_{\alpha} \eta_{3}\right) \mathrm{d} \Gamma_{-}= \\
l^{e b c i}\left(\left(-x_{3} \partial_{1} \eta_{3},-x_{3} \partial_{2} \eta_{3}, 0\right)\right) \quad \forall \eta_{3} \in H^{2}(\omega) \text { with } \eta_{3}=0 \text { on } \gamma_{0} .
\end{aligned}
$$

On the other hand, choosing $\eta_{3}=0$ and $\eta_{\alpha} \neq 0$ for $\alpha=1,2$, i.e., $v=\left(\eta_{1}, \eta_{2}, 0\right)$, (3.36) becomes

$$
\int_{\omega} N_{\alpha \beta}^{e b c i}(u) e_{\alpha \beta}(\eta)+\int_{\Gamma_{-}} \mu_{\alpha} \eta_{\alpha} \mathrm{d} \Gamma_{-}=l^{e b c i}\left(\left(\eta_{1}, \eta_{2}, 0\right)\right) \quad \forall\left(\eta_{1}, \eta_{1}\right) \in\left(H^{1}(\omega)\right)^{2} \text { with } \eta_{\alpha}=0 \text { on } \gamma_{0} \text {. }
$$


Using Green's theorem in (3.37) and (3.38) while neglecting regularity issues, and stating the complementarity conditions satisfied by $(\lambda, \mu)$ leads to the following strong formulation of the limit problem:

Equilibrium equations (coupling mechanical and electric effects)

$$
\left[\begin{array}{l}
\partial_{\alpha \beta} M_{\alpha \beta}^{e b c i}(u)-\lambda+h x_{3} \partial_{\alpha} \mu_{\alpha}=F_{3}^{e b c i} \text { in } \omega, \\
-\partial_{\alpha} N_{\alpha \beta}^{e b c i}(u)+\mu_{\beta}=F_{\beta}^{e b c i} \text { on } \omega, \quad \text { and } \beta=1,2 .
\end{array}\right.
$$

Boundary conditions

$\left[\begin{array}{l}\zeta_{3}=0=\frac{\partial \zeta_{3}}{\partial n}, \quad\left(\zeta_{1}, \zeta_{2}\right)=(0,0) \text { on } \gamma_{0}, \\ \text { boundary conditions for the terms } M_{\alpha \beta}^{e b c i}+\mu_{\alpha} \text { and } N_{\alpha \beta}^{e b c i}\end{array}\right.$.

Constitutive equations

$\left[\begin{array}{l}\sigma_{\alpha \beta}(u, \varphi)=A_{\alpha \beta \gamma \rho} e_{\gamma \rho}(u)+p_{3 \alpha \beta} \partial_{3} \varphi, \quad \sigma_{i 3}(u, \varphi)=0 \quad \text { in } \Omega, \\ D_{i}(u, \varphi)=p_{i \alpha \beta} e_{\alpha \beta}(u)-p_{i 3} \partial_{3} \varphi \quad \text { in } \quad \Omega .\end{array}\right.$

Contact condition

$u_{n}=-u_{3} \leq s, \quad \lambda \geq 0, \quad \lambda\left(u_{n}-s\right)=0 \quad$ on $\quad \Gamma_{-}$.

Friction condition

$$
\left[\begin{array}{l}
|\mu|=\sqrt{\mu_{1}^{2}+\mu_{2}^{2}} \leq q \quad \text { and } \\
|\mu|<q \quad \Rightarrow \quad u_{t}=0, \\
|\mu|=q \quad \Rightarrow \quad \exists c \geq 0: \quad u_{t}=c \mu
\end{array}\right\} \quad \text { on } \Gamma_{-}
$$

The terms $F_{3}^{e b c i}$ and $F_{\beta}^{e b c i}$ represent the transverse and tangential forces acting on the middle plane $\omega$ of the plate. They are related to the mechanical forces, electric data and charges appearing in the definition of the linear form $l^{e b c i}(\cdot)$ (see Part $\mathrm{C}$ of the Appendix for details).

We observe that the limit mechanical displacement $u$ satisfies a system of equations independent of $\varphi$ but depending on the elastic and piezoelectric coefficients as well as the mechanical and electric data and boundary conditions. Moreover, $(u, \varphi)$ also satisfies the limit constitutive equations $(3.39 \mathrm{c})$, which are a consequence of (3.26) and the descalings. In the equations (3.39a), the tangential and transverse displacements $\zeta_{\alpha}$ and $\zeta_{3}$ are coupled due to the anisotropy of the material (as can be seen in the definitions of $M_{\alpha \beta}^{e b c i}(u)$ and $N_{\alpha \beta}^{e b c i}(u)$ ) and since to the friction condition (the Lagrange multiplier $\mu$ appears in both equations of (3.39a)).

For the case of a homogeneous and isotropic material and if we neglect friction, the tangential and transverse displacements $\zeta_{\alpha}$ and $\zeta_{3}$ decouple in the asymptotic model. This happens since the equations in (3.39a) become independent from each other since the friction terms containing $\mu$ vanish, since $M_{\alpha \beta}^{e b c i}(u)=M_{\alpha \beta}^{e b c i}\left(\zeta_{3}\right)$ only depends on $\zeta_{3}$ and $N_{\alpha \beta}^{e b c i}(u)=N_{\alpha \beta}^{e b c i}\left(\zeta_{1}, \zeta_{2}\right)$ only depends on $\left(\zeta_{1}, \zeta_{2}\right)$, see also [8,11].

Remark 3.6. The derivations in this paper remain true for two special cases: Firstly, for bilateral contact problems (i.e., the contact region is known a priori) with Tresca friction. And, secondly, for unilateral contact without friction. The latter case corresponds, at least formally, to the choice $q \equiv 0$. Both of these special cases are physically more meaningful than unilateral (i.e., unknown) contact with Tresca friction. However, for the realization of contact with Coulomb friction, which is realistic and often used in practice, usually a sequence of Tresca frictional contact problems is solved. This renders unilateral contact with Tresca friction to an important problem as well. 
It should be mentioned that the asymptotic procedure employed in this paper applies in a similar way to contact problems with thin linear elastic plates with integrated piezoelectric patches or layers. The corresponding asymptotic models can be derived using similar arguments as in this paper, provided the piezoelectric patches or layers are perfectly linked (surface bonded or embedded) to the elastic plates.

\section{NumericAl EXAMPLES}

Here, we present numerical tests for the asymptotic equations obtained in the previous sections. We first use a simple example to verify our code and to discuss the friction and contact conditions and then focus on two examples, where the mechanical frictional contact behavior interacts significantly with the electric potential. The numerical treatment of the contact and friction conditions for the mechanical displacement follows [16]. The mechanical equations are discretized with bilinear finite elements for the tangential components $\zeta_{1}$ and $\zeta_{2}$ and with third-order nonconforming elements for $\zeta_{3}$. Note that, while the asymptotic equations are defined on a two-dimensional domain, the contact and friction conditions remain structurally as in three-dimensional contact mechanics, where frictional contact occurs on two-dimensional boundary surfaces. To simplify the problem we replace for our numerical implementation the contact and friction conditions on $\Gamma_{-}$by the same conditions in the middle plane (and, for consistency, we also elevate the obstacle by $h$ ).

In all our examples we consider a laminated plate made of two layers of different PZT piezoelectric ceramic materials. The material parameters are taken from the Tables VIII and XI in [19]. Both layers are assumed to be of the same thickness $h=0.01$ leading to a plate of thickness 0.02 . The data are given in SI units, i.e., length is measured in meter, mechanical forces in Newton and electrical potentials in Volt.

\subsection{Example 1}

Using this example we verify our implementation for the contact conditions and briefly discuss properties of Tresca friction contact problems. We assume a plate with middle plane $\omega=[0,1] \times[0,2]$ that is subject to the mechanical volume forces $f \equiv\left(5 \times 10^{7}, 0,-5 \times 10^{5}\right)$. The obstacle is given by $s=0.015$ and we assume the case $(e b c 2)$ with all zero electrical boundary conditions. The friction bound for the Tresca friction law is $q=10^{7}$. In the upper plot of Figure 2 we show the deformed plate. In the lower left plot, the a priori unknown contact nodes are shown as black dots. In the lower right plot of Figure 2 we visualize the tangential stress (red arrows) and the tangential displacement (blue arrows). Note that sliding occurs only in direction of the tangential stress as required in the Tresca friction law. Moreover, note that friction (that is, nonzero tangential stress) occurs also in points where the plate is not in contact with the obstacle, which is an unphysical behavior. This is due to the fact that in the Tresca friction law an a priori given friction bound $q$ is used, see also Remark 3.6. The more realistic Coulomb friction law uses $q=\mathcal{F}\left|\sigma_{n}\right|$ with the so called friction coefficient $\mathcal{F} \geq 0$. Thus, in the latter case, $q$ depends on $u$ (see also Ex. 2). For a more detailed discussion of friction laws we refer to [9,13], see also [16].

\subsection{Example 2}

For this second example, $\omega=[0,1] \times[0,2]$ and we again use the electric boundary conditions $(e b c 2)$. To be more precise, we apply an electric surface charge density $\theta=3$ on the upper surface $\Gamma_{+}$and assume the lower surface to be grounded. Moreover, all mechanical forces are zero with exception of $f_{3} \equiv-10^{5}$ (that is, the plate is subject to a gravity force pressing it onto the obstacle). In Figure 3, we show the deformed plate using various different obstacles. Besides the Signorini contact conditions, we use the Coulomb friction law since it is more realistic than the Tresca law. Coulomb friction means that $q$ is not given a priori, but that $q=\mathcal{F}\left|\sigma_{n}\right|$, with $\mathcal{F} \geq 0$ (we use $\mathcal{F}=1$ ). The Coulomb friction problem is numerically treated by solving a sequence of Tresca friction problems, see [16]. The computations shown in Figure 3 are done using $40 \times 80$ finite elements.

Remarkably, the region of actual contact between obstacle and plate is relatively small, even though additionally to the applied electric surface charge density the plate is subject to a gravity force pressing it towards the obstacle: For all obstacles, contact essentially only occurs along one-dimensional curves consisting of relatively 

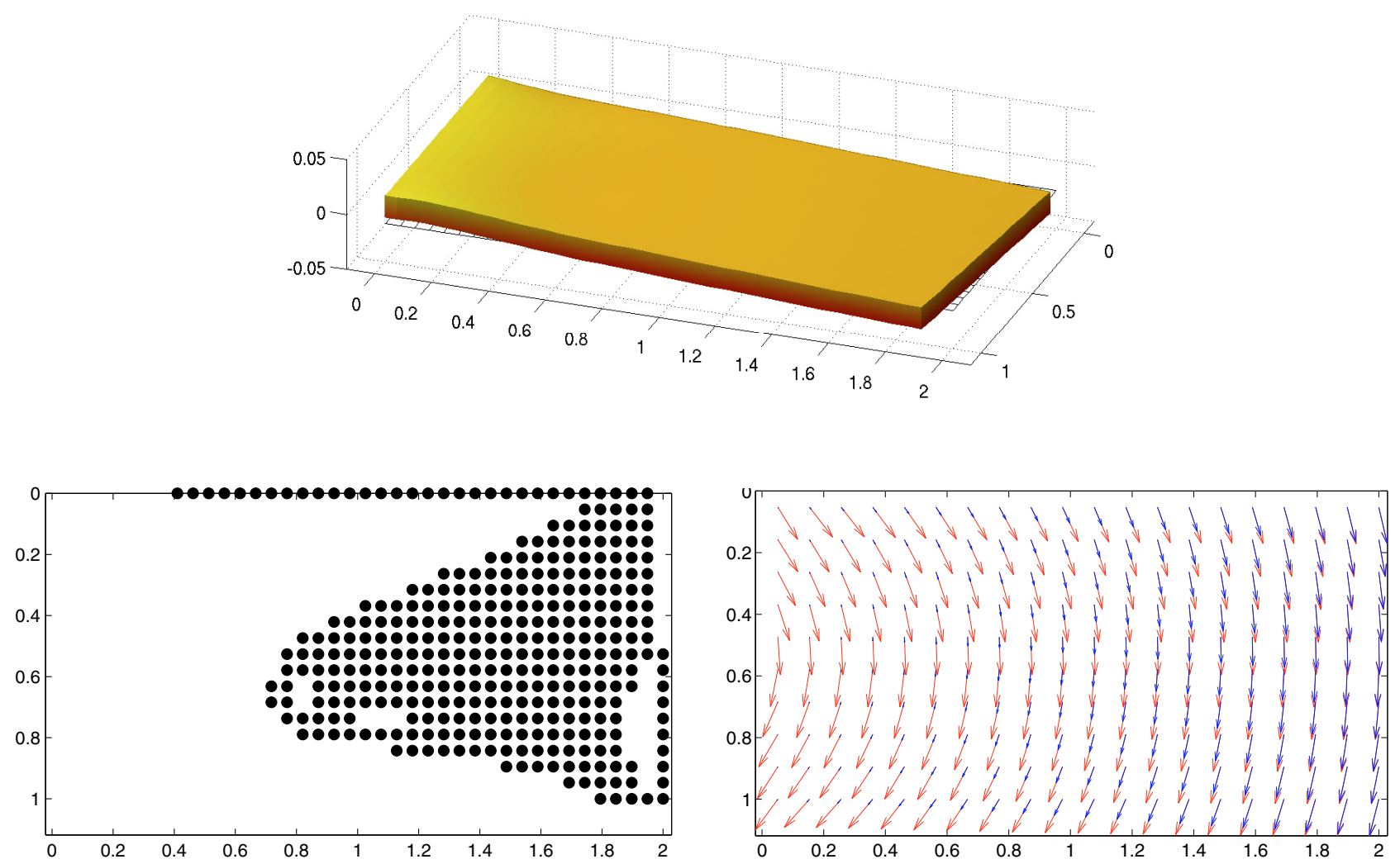

Figure 2. Example 1: deformed plate (upper plot); top view of nodes where the plate is in contact with the rigid obstacle (black dots in lower left plot); top view of tangential displacement (blue) and tangential stress (red, lower right plot).

few points. This remains true when the mesh is refined to, e.g., to $80 \times 160$ elements. A possible explanation for this behavior is that large inner stresses resulting from the applied electric surface charge density $\theta$ avoid a larger contact region with the obstacle. We have observed a similar phenomenon also for the Tresca friction law and for other (non affine) obstacles: If an electric surface charge is applied to the plate, contact regions are remarkably small.

\subsection{Example 3}

With this last example we show, how the direct piezoelectric effect can be used for the detection and study of obstacles. We use a plate with middle plane $\omega=[0,1] \times[0,1]$ that is clamped along the boundary $\{0\} \times[0,1]$. It is subject to the constant gravity force $f_{3}=-10^{6}$. All other mechanical forces are zero and there are no applied electrical forces. We use the boundary conditions $(e b c 2)$ with grounded lower surface $\left(\varphi_{0}=0\right.$ on $\left.\Gamma_{-}\right)$. On the upper surface $\Gamma_{+}$, where we measure the electric potential, we assume homogeneous electric Neumann boundary conditions. The plate deforms from its original state due to the applied mechanical force and the shape of the obstacle. For this example we neglect the frictional forces and only assume Signorini contact conditions. We focus on the electric potential on the upper surface of the plate and study if it is possible to draw conclusions about the shape of the obstacle. 

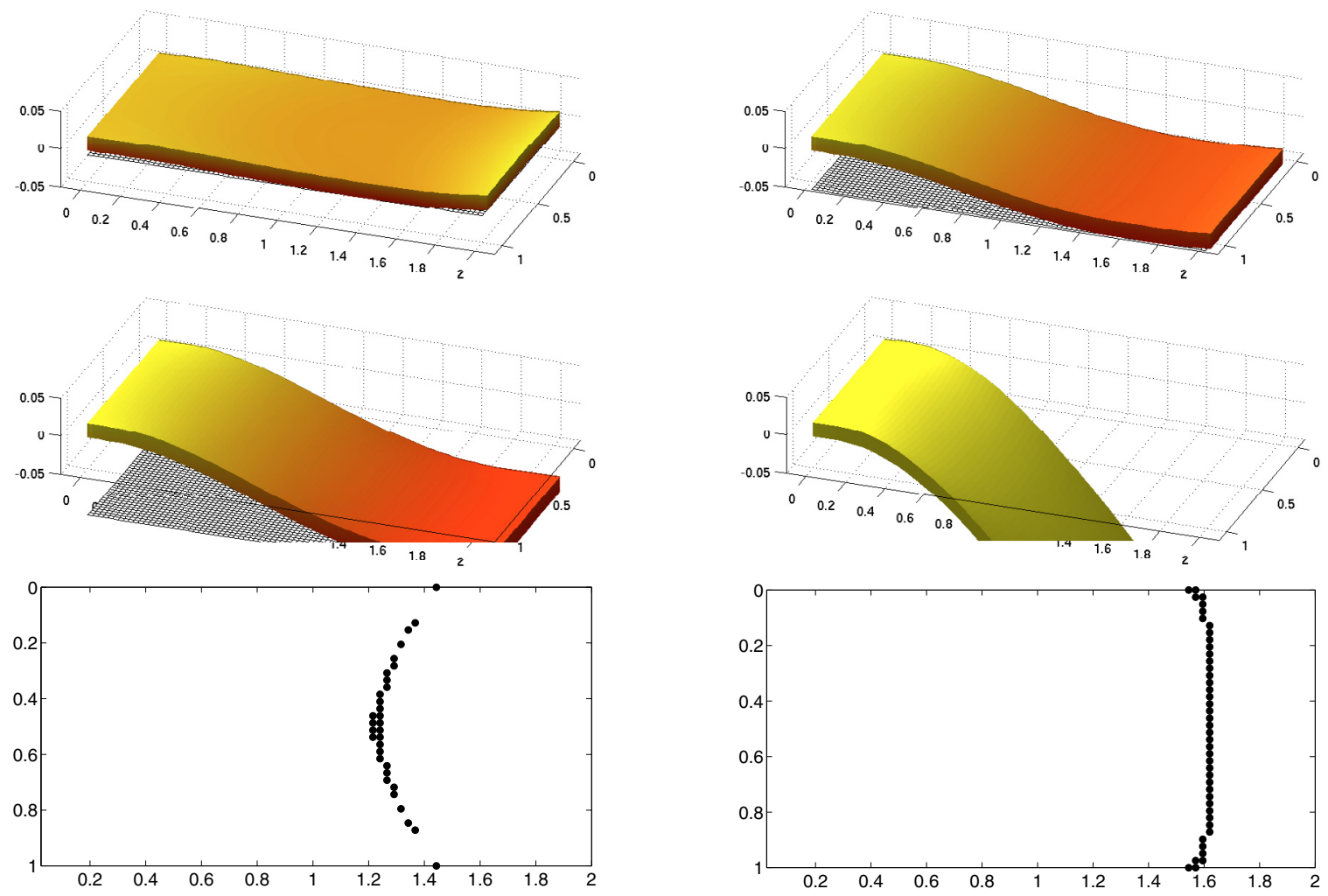

Figure 3. Example 2: deformed plate subject to contact with Coulomb friction, i.e. $q=\left|\sigma_{n}\right|$; obstacle corresponding to $s=0.015$ (upper left), to $s=0.06$ (upper right), to $s=0.11$ (middle left) and deformation if not restricted with an obstacle (middle right); top view of contact nodes for $s=0.015$ (lower, left) and for $s=0.06$ (lower, right) - note that the contact regions are small.

In Figure 4 we show the three different obstacles used (left column), the corresponding deformed plates (middle) and the contour plots of the electric potentials on $\Gamma_{+}$(right). Note that the electric potential on the upper surface allows to guess the shape of the obstacle. In this sense, piezoelectric plates can be used as sensors for scanning and for the study of surfaces.

\section{APPENDIX}

Part A. The modified tensors $A_{\alpha \beta \gamma \rho}, p_{i \alpha \beta}$ and $p_{i 3}$, in Theorem 3.1, are defined by

$$
\begin{aligned}
& A_{\alpha \beta \gamma \rho}:=C_{\alpha \beta \gamma \rho}-\frac{C_{\alpha \beta 33} C_{33 \gamma \rho}}{C_{3333}}+\left(C_{\alpha \beta 33} \frac{C_{\nu 333}}{C_{3333}}-C_{\alpha \beta \nu 3}\right) b_{\delta \nu} a_{\delta \gamma \rho}, \\
& p_{i \alpha \beta}:=P_{i \alpha \beta}-\frac{C_{\alpha \beta 33}}{C_{3333}} P_{i 33}+\left(P_{i 33} \frac{C_{33 \nu 3}}{C_{3333}}-P_{i \nu 3}\right) b_{\delta \nu} a_{\delta \alpha \beta} \\
& p_{i 3}:=\varepsilon_{i 3}+\frac{P_{i 33} P_{333}}{C_{3333}}-\left(P_{i 33} \frac{C_{33 \nu 3}}{C_{3333}}-P_{i \nu 3}\right) b_{\delta \nu} c_{\delta}
\end{aligned}
$$



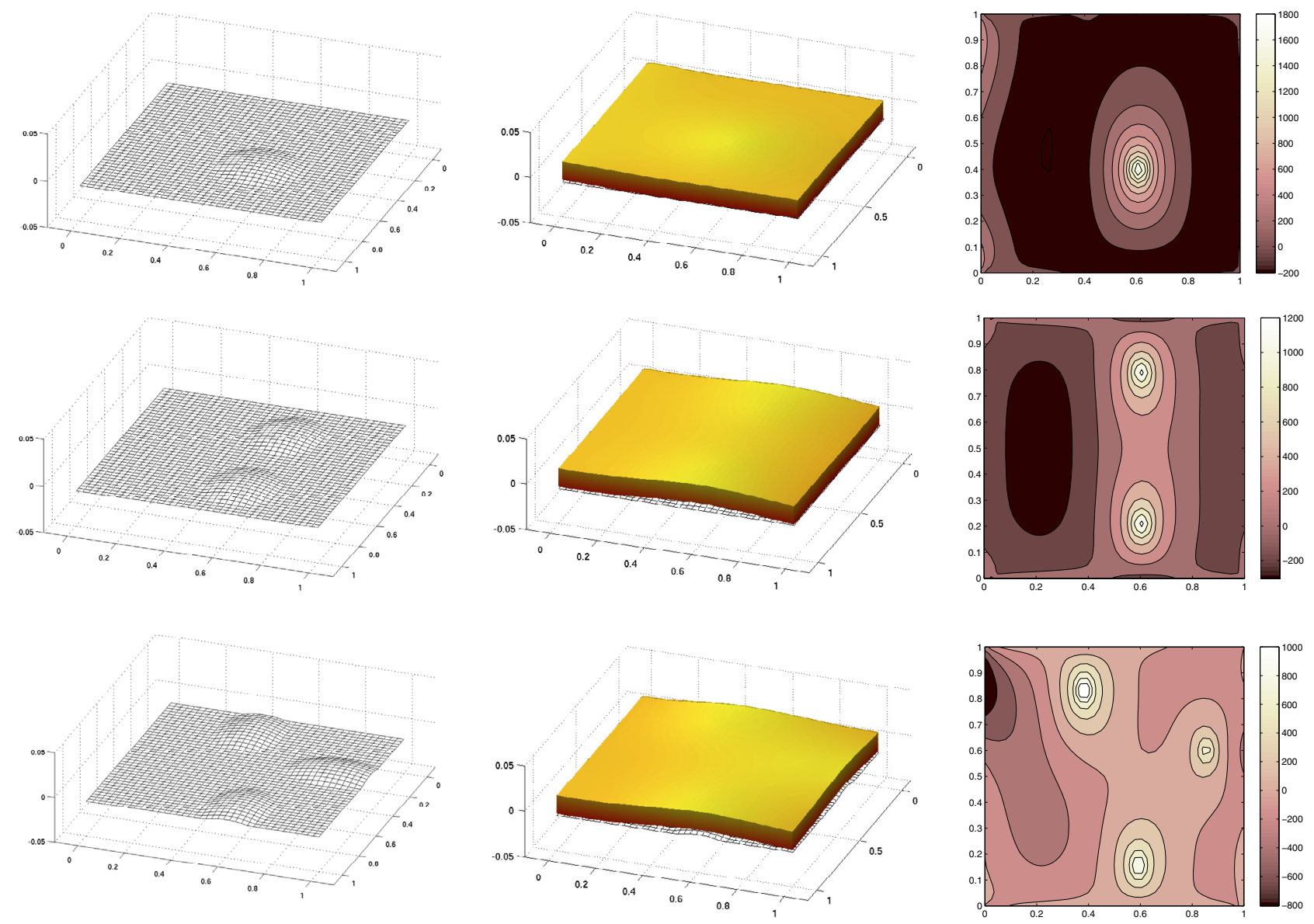

FigurE 4. Example 3: different obstacles (left), the corresponding deformed plates (middle) and contour plots of the electric potentials on $\Gamma_{+}$(right).

where

$$
\begin{aligned}
& a_{\delta \gamma \rho}:=C_{33 \gamma \rho} C_{\delta 333}-C_{\delta 3 \gamma \rho} C_{3333}, \quad c_{\delta}:=C_{\delta 333} P_{333}-C_{3333} P_{3 \delta 3}, \\
& {\left[b_{\delta \nu}\right]:=\left[C_{\delta 333} C_{33 \nu 3}-C_{\delta 3 \nu 3} C_{3333}\right]^{-1} \quad \text { (identity between two matrices). }}
\end{aligned}
$$

We remark that $p_{3 \alpha \beta}$ can equivalently be computed as

$$
p_{3 \alpha \beta}:=P_{3 \alpha \beta}-\frac{C_{\alpha \beta 33}}{C_{3333}} P_{333}+\left(C_{\alpha \beta 33} \frac{C_{33 \nu 3}}{C_{3333}}-C_{\alpha \beta \nu 3}\right) b_{\delta \nu} c_{\delta} .
$$

Part B. The terms $\left(N_{\alpha \beta}^{e b c i}(u)\right)$ and $\left(M_{\alpha \beta}^{e b c i}(u)\right)$, in Theorem 3.3, are the components of second-order tensor fields corresponding to the Kirchhoff-Love displacement $u$, given by the following matrix formula

$$
\left[\begin{array}{c}
N_{\alpha \beta}^{e b c i}(u) \\
M_{\alpha \beta}^{e b c i}(u)
\end{array}\right]=O^{e b c i}\left[\begin{array}{c}
e_{\gamma \rho}(\zeta) \\
\partial_{\gamma \rho} \zeta_{3}
\end{array}\right]
$$


where the components of the $6 \times 6$ matrix $O^{e b c i}$ are functions of the middle plane $\omega$, namely

$$
O^{e b c i}=\left[\begin{array}{cc}
\int_{-h}^{+h} C_{\alpha \beta \gamma \rho}^{e b c i} \mathrm{~d} x_{3} & -\int_{-h}^{+h} D_{\alpha \beta \gamma \rho}^{e b c i} \mathrm{~d} x_{3} \\
-\int_{-h}^{+h} x_{3} C_{\alpha \beta \gamma \rho}^{e b c i} \mathrm{~d} x_{3} & \int_{-h}^{+h} x_{3} D_{\alpha \beta \gamma \rho}^{e b c i} \mathrm{~d} x_{3}
\end{array}\right]_{6 \times 6},
$$

with the modified coefficients defined on $\Omega$ :

$$
\begin{aligned}
& B_{\alpha \beta \gamma \rho}:=A_{\alpha \beta \gamma \rho}+\frac{p_{3 \alpha \beta} p_{3 \gamma \rho}}{p_{33}} \\
& C_{\alpha \beta \gamma \rho}^{e b c i}:=B_{\alpha \beta \gamma \rho}+\tilde{C}_{\alpha \beta \gamma \rho}^{e b c i} \quad \text { with } \tilde{C}_{\alpha \beta \gamma \rho}^{e b c i}=\left\{\begin{array}{cl}
-\frac{p_{3 \alpha \beta} a_{\gamma \rho}}{p_{33}} c_{0} & \text { for }(e b c 1), \\
0 & \text { for }(e b c 2) \text { and }(e b c 3),
\end{array}\right. \\
& D_{\alpha \beta \gamma \rho}^{e b c i}:=x_{3} B_{\alpha \beta \gamma \rho}+\tilde{D}_{\alpha \beta \gamma \rho}^{e b c i} \quad \text { with } \tilde{D}_{\alpha \beta \gamma \rho}^{e b c i}=\left\{\begin{array}{cl}
-\frac{p_{3 \alpha \beta} b_{\gamma \rho}}{p_{33}} c_{0} & \text { for }(e b c 1), \\
0 & \text { for }(e b c 2) \text { and }(e b c 3) .
\end{array}\right.
\end{aligned}
$$

The linear form $l^{e b c i}($.$) is defined by$

$$
l^{e b c i}(v):=\int_{\Omega} f \cdot v \mathrm{~d} \omega+\int_{\Gamma_{N}} g \cdot v \mathrm{~d} \Gamma_{N}+l_{e}^{e b c i}(v),
$$

with

$$
l_{e}^{e b c i}(v):=\left\{\begin{array}{l}
\int_{\Omega}\left(P_{3} r+\left(\varphi_{0}^{-}-\varphi_{0}^{+}-R\right) c_{0}\right) \frac{p_{3 \alpha \beta}}{p_{33}} e_{\alpha \beta}(v) \mathrm{d} x \quad \text { for }(e b c 1), \\
\left\{\begin{array}{l}
\int_{\Omega} \frac{p_{3 \alpha \beta}}{p_{33}}\left(P_{3} r\left(x_{1}, x_{2}, x_{3}\right)+\left(h^{*} \theta-P_{3} r\right)\left(x_{1}, x_{2}, h^{*}\right)\right) e_{\alpha \beta}(v), \\
\text { with } h^{*}=+1 \text { for }(e b c 2) \text { and } h^{*}=-1 \text { for }(e b c 3) .
\end{array}\right.
\end{array}\right.
$$

Part C. The formulas for $F_{3}^{e b c i}$ and $F_{\beta}^{e b c i}$, which appear in Remark 3.5, are defined by

$$
\begin{aligned}
& F_{3}^{e b c i}=\int_{-h}^{+h}\left(x_{3} \partial_{\alpha} f_{\alpha}+f_{3}\right) \mathrm{d} x_{3}+g_{3}^{+}+g_{3}^{-}+h \partial_{\alpha}\left(g_{\alpha}^{+}-g_{\alpha}^{-}\right)+\partial_{\alpha \beta}\left(-x_{3} G_{\alpha \beta}^{e b c i}\right), \\
& F_{\beta}^{e b c i}=\int_{-h}^{+h} f_{\beta} \mathrm{d} x_{3}+\left(g_{\beta}^{+}+g_{\beta}^{-}\right)-\partial_{\alpha} G_{\alpha \beta}^{e b c i} \quad \text { for } \quad \beta=1,2,
\end{aligned}
$$

where

$$
G_{\alpha \beta}^{e b c i}=\left\{\begin{array}{l}
\int_{-h}^{+h}\left(P_{3} r+\left(\varphi_{0}^{-}-\varphi_{0}^{+}-R\right) c_{0}\right) \frac{p_{3 \alpha \beta}}{p_{33}} \mathrm{~d} x_{3} \quad \text { for }(e b c 1) \\
\left\{\begin{array}{l}
\int_{-h}^{+h} \frac{p_{3 \alpha \beta}}{p_{33}}\left(P_{3} r\left(x_{1}, x_{2}, x_{3}\right)+\left(h^{*} \theta-P_{3} r\right)\left(x_{1}, x_{2}, h^{*}\right)\right) \mathrm{d} x_{3}, \\
\text { with } h^{*}=+1, \text { if } i=2 \text { and } h^{*}=-1, \text { if } i=3 .
\end{array}\right.
\end{array}\right.
$$

\section{REFERENCES}

[1] M. Bernadou and C. Haenel, Modelization and numerical approximation of piezoelectric thin shells. I. The continuous problems. Comput. Methods Appl. Mech. Engrg. 192 (2003) 4003-4043.

[2] P. Bisegna, F. Lebon and F. Maceri, The unilateral frictional contact of a piezoelectric body with a rigid support, in Contact mechanics (Praia da Consolação, 2001), Solid Mech. Appl., Kluwer Acad. Publ., Dordrecht (2002) 347-354.

[3] P.G. Ciarlet, Mathematical Elasticity, Vol. II: Theory of Plates, Studies in Mathematics and its Applications 27. NorthHolland Publishing Co., Amsterdam (1997).

[4] P.G. Ciarlet, Mathematical Elasticity. Vol. III: Theory of Shells, Studies in Mathematics and its Applications 29. NorthHolland Publishing Co., Amsterdam (2000). 
[5] P.G. Ciarlet and P. Destuynder, Une justification d'un modèle non linéaire en théorie des plaques. C. R. Acad. Sci. Paris Sér. A-B 287 (1978) A33-A36.

[6] P.G. Ciarlet and P. Destuynder, A justification of the two-dimensional linear plate model. J. Mécanique 18 (1979) 315-344.

[7] C. Collard and B. Miara, Two-dimensional models for geometrically nonlinear thin piezoelectric shells. Asymptotic Anal. 31 (2002) 113-151.

[8] L. Costa, I. Figueiredo, R. Leal, P. Oliveira and G. Stadler, Modeling and numerical study of actuator and sensor effects for a laminated piezoelectric plate. Comput. Struct. 85 (2007) 385-403.

[9] G. Duvaut and J.-L. Lions, Inequalities in Mechanics and Physics, Grundlehren der mathematischen Wissenschaften 219. Springer-Verlag, Berlin (1976).

[10] I. Ekeland and R. Temam, Convex Analysis and Variational Problems, Classics in Applied Mathematics 28. SIAM, Philadelphia (1999).

[11] I. Figueiredo and C. Leal, A piezoelectric anisotropic plate model. Asymptotic Anal. 44 (2005) 327-346.

[12] I. Figueiredo and C. Leal, A generalized piezoelectric Bernoulli-Navier anisotropic rod model. J. Elasticity 85 (2006) $85-106$.

[13] R. Glowinski, Numerical Methods for Nonlinear Variational Inequalities. Springer-Verlag, New York (1984).

[14] J. Haslinger, M. Miettinen and P. Panagiotopoulos, Finite Element Method for Hemivariational Inequalities, Nonconvex Optimization and its Applications 35. Kluwer Academic Publishers, Dordrecht (1999).

[15] S. Hüeber, A. Matei and B.I. Wohlmuth, A mixed variational formulation and an optimal a priori error estimate for a frictional contact problem in elasto-piezoelectricity. Bull. Math. Soc. Sci. Math. Roumanie (N.S.) 48 (2005) 209-232.

[16] S. Hüeber, G. Stadler and B. Wohlmuth, A primal-dual active set algorithm for three-dimensional contact problems with Coulomb friction. SIAM J. Sci. Comp. 30 (2008) 572-596.

[17] T. Ikeda, Fundamentals of Piezoelectricity. Oxford University Press (1990).

[18] N. Kikuchi and J.T. Oden, Contact Problems in Elasticity: A Study of Variational Inequalities and Finite Element Methods, SIAM Studies in Applied Mathematics 8. Society for Industrial and Applied Mathematics (SIAM), Philadelphia, PA (1988).

[19] S. Klinkel and W. Wagner, A geometrically non-linear piezoelectric solid shell element based on a mixed multi-field variational formulation. Int. J. Numer. Meth. Engng. 65 (2005) 349-382.

[20] A. Léger and B. Miara, Mathematical justification of the obstacle problem in the case of a shallow shell. J. Elasticity 9 (2008) $241-257$.

[21] J.-L. Lions, Perturbations singulières dans les problèmes aux limites et en contrôle optimal, Lecture Notes in Mathematics 323. Springer-Verlag, Berlin (1973).

[22] F. Maceri and P. Bisegna, The unilateral frictionless contact of a piezoelectric body with a rigid support. Math. Comput. Model. 28 (1998) 19-28.

[23] G.A. Maugin and D. Attou, An asymptotic theory of thin piezoelectric plates. Quart. J. Mech. Appl. Math. 43 (1990) $347-362$.

[24] B. Miara, Justification of the asymptotic analysis of elastic plates. I. The linear case. Asymptotic Anal. 9 (1994) 47-60.

[25] M. Rahmoune, A. Benjeddou and R. Ohayon, New thin piezoelectric plate models. J. Int. Mat. Sys. Struct. 9 (1998) $1017-1029$.

[26] A. Raoult and A. Sène, Modelling of piezoelectric plates including magnetic effects. Asymptotic Anal. 34 (2003) 1-40.

[27] N. Sabu, Vibrations of thin piezoelectric flexural shells: Two-dimensional approximation. J. Elast. 68 (2002) 145-165.

[28] A. Sene, Modelling of piezoelectric static thin plates. Asymptotic Anal. 25 (2001) 1-20.

[29] R.C. Smith, Smart Material Systems: Model Development, Frontiers in Applied Mathematics 32. Society for Industrial and Applied Mathematics (SIAM), Philadelphia, PA (2005).

[30] M. Sofonea and El-H. Essoufi, A piezoelectric contact problem with slip dependent coefficient of friction. Math. Model. Anal. 9 (2004) 229-242.

[31] M. Sofonea and El-H. Essoufi, Quasistatic frictional contact of a viscoelastic piezoelectric body. Adv. Math. Sci. Appl. 14 (2004) 613-631.

[32] L. Trabucho and J.M. Viaño, Mathematical modelling of rods, in Handbook of Numerical Analysis IV, P.G. Ciarlet and J.-L. Lions Eds., Elsevier, Amsterdam, North-Holland (1996) 487-974.

[33] T. Weller and C. Licht, Analyse asymptotique de plaques minces linéairement piézoélectriques. C. R. Math. Acad. Sci. Paris 335 (2002) 309-314. 